\title{
BMJ Open Early detection of Australian Aboriginal and Torres Strait Islander infants at high risk of adverse neurodevelopmental outcomes at 12 months corrected age: LEAP-CP prospective cohort study protocol
}

\author{
Carly R Luke (1) , ${ }^{1,2}$ Katherine Benfer, ${ }^{1}$ Leeann Mick-Ramsamy, ${ }^{1}$ \\ Robert S Ware (D) , ${ }^{3}$ Natasha Reid, ${ }^{4}$ Arend F Bos, ${ }^{5}$ Margot Bosanquet, ${ }^{2}$ \\ Roslyn N Boyd ${ }^{1}$
}

To cite: Luke CR, Benfer K, Mick-Ramsamy L, et al. Early detection of Australian Aboriginal and Torres Strait Islander infants at high risk of adverse neurodevelopmental outcomes at 12 months corrected age: LEAP-CP prospective cohort study protocol. BMJ Open 2022;12:e053646. doi:10.1136/ bmjopen-2021-053646

- Prepublication history and additional supplemental material for this paper are available online. To view these files, please visit the journal online (http://dx.doi.org/10.1136/ bmjopen-2021-053646).

Received 19 May 2021 Accepted 10 December 2021

Check for updates

(C) Author(s) (or their employer(s)) 2022. Re-use permitted under CC BY-NC. No commercial re-use. See rights and permissions. Published by BMJ.

For numbered affiliations see end of article.

Correspondence to

Carly R Luke;

Carly.Luke@uqconnect.edu.au

\section{ABSTRACT}

Introduction Neurodevelopmental disorders (NDD), including cerebral palsy (CP), autism spectrum disorder (ASD) and foetal alcohol spectrum disorder (FASD), are characterised by impaired development of the early central nervous system, impacting cognitive and/or physical function. Early detection of NDD enables infants to be fast-tracked to early intervention services, optimising outcomes. Aboriginal and Torres Strait Islander infants may experience early life factors increasing their risk of neurodevelopmental vulnerability, which persist into later childhood, further compounding the health inequities experienced by First Nations peoples in Australia. The LEAP-CP prospective cohort study will investigate the efficacy of early screening programmes, implemented in Queensland, Australia to earlier identify Aboriginal and Torres Strait Islander infants who are 'at risk' of adverse neurodevelopmental outcomes (NDO) or NDD. Diagnostic accuracy and feasibility of early detection tools for identifying infants 'at risk' of a later diagnosis of adverse NDO or NDD will be determined.

Methods and analysis Aboriginal and/or Torres Strait Islander infants born in Queensland, Australia (birth years 2020-2022) will be invited to participate. Infants aged $\leq 9$ months corrected age (CA) will undergo screening using the (1) General Movements Assessment (GMA); (2) Hammersmith Infant Neurological Examination (HINE); (3) Rapid Neurodevelopmental Assessment (RNDA) and (4) Ages and Stages Questionnaire-Aboriginal adaptation (ASQ-TRAK). Developmental outcomes at 12 months CA will be determined for: (1) neurological (HINE); (2) motor (Peabody Developmental Motor Scales 2); (3) cognitive and communication (Bayley Scales of Infant Development III); (4) functional capabilities (Paediatric Evaluation of Disability Inventory-Computer Adaptive Test) and (5) behaviour (Infant Toddler Social and Emotional Assessment). Infants will be classified as typically developing or 'at risk' of an adverse NDO and/or specific NDD based on symptomology using developmental and diagnostic outcomes for (1) CP (2) ASD and (3) FASD. The effects of perinatal, social and
Strengths and limitations of this study

- This prospective population-based cohort study investigates the use of standardised screening tools to predict a later diagnosis of adverse neurodevelopmental outcomes in an Australian Aboriginal and Torres Strait Islander birth cohort.

- Capacity building of local services and use of technology ensures infants and families can readily access gold standard screening programmes close to home.

- Community and stakeholder engagement, knowledge sharing and codesign promotes access to culturally sensitive programmes.

- The remote locality of many Indigenous communities in Australia may present challenges, limiting access to health services and impacting loss to follow-up of infants at study outcome timelines.

environmental factors, caregiver mental health and clinical neuroimaging on NDOs will be investigated.

Ethics and dissemination Ethics approval has been granted by appropriate Queensland ethics committees; Far North Queensland Health Research Ethics Committee (HREC/2019/QCH/50533 (Sep ver 2)-1370), the Townsville HHS Human Research Ethics Committee (HREC/ QTHS/56008), the University of Queensland Medical Research Ethics Committee (2020000185/HREC/2019/ QCH/50533) and the Children's Health Queensland HHS Human Research Ethics Committee (HREC/20/QCHQ/63906) with governance and support from local First Nations communities. Findings from this study will be disseminated via peer-reviewed publications and conference presentations. Trial registration number ACTRN12619000969167.

\section{INTRODUCTION}

In Australia, Aboriginal and Torres Strait Islander peoples are among the most 
disadvantaged across all domains. In acknowledgement of the unique and distinct countries, cultures and languages of Australian First Nations people, the term 'Indigenous' is respectfully used herein to encompass but not homogenise the diverse identities of Aboriginal and Torres Strait Islander peoples.

Ongoing intergenerational trauma, systematic displacement from traditional lands, loss of culture and racism experienced by Australian Indigenous people continues to manifest in socioeconomic disadvantage, marginalisation, reduced education and employment opportunities, leading to poorer health outcomes. ${ }^{12}$ Indigenous Australians are 1.8 times more likely to experience disability, twice as likely to have a severe disability and are less likely to access support ${ }^{3}$ compared with non-indigenous Australians. ${ }^{4}{ }^{5}$ Inequities in access to culturally safe health and disability support services, ${ }^{6}$ long waiting lists and the rurality of some Indigenous communities, further compounds this disadvantage. ${ }^{78}$ These factors have contributed to a significant gap in health outcomes between Indigenous and non-Indigenous Australians. ${ }^{39}$

Indigenous children, living in urban, rural and remote Australia, have an increased risk of adverse neurodevelopmental outcomes (NDO). This can include being at risk for a range of specific childhood neurodevelopmental disorders (NDD): cerebral palsy (CP), foetal alcohol spectrum disorder (FASD) and autism spectrum disorder (ASD). ${ }^{81011}$ These conditions are characterised by impaired development of the early central nervous system, resulting in cognitive and/or physical disability. ${ }^{12}{ }^{13}$ Indigenous children are $30 \%$ more likely to have a physical disability, and are at higher risk of developmental and intellectual difficulties, compared with non-Indigenous children. ${ }^{11} 1415$ The prevalence of NDDs in some remote communities is reported to be as high as $30 \%$ of the paediatric population. ${ }^{10}$

\section{Indigenous infant early life risk factors}

Many Australian Indigenous infants can experience a range of perinatal, maternal, postneonatal (PNN) and socioeconomic risk factors that increase their risk of later adverse NDOs. While the neonatal death rate for Indigenous infants has declined, the rates of preterm birth (ie, $<37$ weeks GA), low birth weight (LBW; ie, $<2500 \mathrm{~g}$ ) and small for gestational age (SGA) births have remained relatively stable. ${ }^{16}$ In 2018, infants of Indigenous mothers were $65 \%$ more likely to be born preterm, $87 \%$ more likely to be LBW and $52 \%$ more likely to be SGA, compared with babies of non-Indigenous mothers. ${ }^{16}$ In addition, $28 \%$ of Indigenous infants were admitted to the Neonatal Intensive Care Unit (NICU) or Special Care Nursery (SCN), requiring specialised medical treatment. ${ }^{16}$

Improving Indigenous birth outcomes, including preterm birth and LBW, is a national priority for the Australian Closing the Gap Agenda. ${ }^{17}$ Infants born preterm and with LBW have an increased risk of adverse NDOs, which can influence school readiness and academic achievement. ${ }^{18-22}$ Biological and environmental risk factors impact birth outcomes and are associated with increased risk of developmental vulnerability. ${ }^{1423-25}$ These factors are compounded by remote locality, access to appropriate and culturally sensitive antenatal care, and, socioeconomic disadvantage. ${ }^{23-25}$ Maternal factors including age, education, health, smoking and substance use have been linked to poorer birth outcomes. ${ }^{142425}$ In Australia, Indigenous mothers are more likely to be younger, single, attain lower levels of education, live in lower socioeconomic circumstances and have lower rates of attendance at antenatal care. ${ }^{1625}$ Emerging evidence demonstrates the protective impact of culturally led ${ }^{26}$ birthing programmes which have led to an improved uptake in antenatal care and smoking cessation, subsequently lowering the risk of neonatal and adverse developmental outcomes. ${ }^{26-29}$

The cultural, geographical and socioeconomic barriers to healthcare access experienced by Indigenous Australians can lead to delayed identification of infants at risk of adverse NDOs with subsequent delays in receiving early intervention to optimise outcomes. ${ }^{11}{ }^{30}$ While there is consensus that early detection is important for all adverse NDOs, variability exists in the recommendations for the screening and diagnosis of CP, ASD and FASD.

\section{Neurodevelopmental disorders}

NDDs are characterised by distinct clinical manifestations and symptomology. A transdiagnostic approach supports the notion that many NDDs share similar early markers and comorbidities across multiple neurodevelopmental domains. ${ }^{31-33}$ Targeted early screening programmes should aim to identify an infant's risk status for a range of adverse NDOs which may predict a later specific diagnosis. ${ }^{32} 34$ Differences in quality of movement, atypical motor development and cognition are common early risk attributes and neurodevelopmental features of CP, ASD and FASD. ${ }^{10}{ }^{35-39}$ We hypothesise that valid and reliable predictive tools utilised for the detection of CP may also identify early neurodevelopmental vulnerabilities in infants at risk of a later diagnosis of ASD and FASD and/ or other substantial developmental delays.

\section{Cerebral palsy}

Cerebral palsy, the most common physical disability of childhood ( 1 in 700 live births), ${ }^{40}$ is defined as a developmental disorder of movement and posture attributed to non-progressive disturbances in the developing brain that occur in early infancy, impacting function, participation and self-care. ${ }^{41}$ Injury to the developing brain can occur preneonatally, perineonatally or PNN due to a recognised event associated with brain damage. ${ }^{8}$

Improvements in medical care and neuroprotective interventions for preterm birth, LBW and other pregnancy complications have been associated with a decline in the overall rate of $\mathrm{CP}^{42}$ Advances in early detection, diagnosis, prevention and intervention in high resource countries have additionally led to improvements in CP prognosis and decreased incidence. ${ }^{42}{ }^{43}$ In Australia, the 
trend in declining $\mathrm{CP}$ rates has demonstrated a decrease in incidence from 1 in 500 children to 1 in 700 children and a reduction in severity of motor function, with more children ambulant. ${ }^{40} 43$

International Clinical Practice Guidelines support a confirmed or 'high risk' of CP diagnosis prior to 6 months $\mathrm{CA} ;{ }^{44}$ however, the age of diagnosis of CP in high income countries still occurs relatively late, usually between 12 and 24 months, delaying access to early intervention services. ${ }^{44}$ The use of gold standard clinical assessments, such as Prechtl's Qualitative Assessment of General Movements (GMA), the Hammersmith Infant Neurological Examination (HINE) and MRI, is recommended for reliable and accurate prediction of 'high risk' of CP. ${ }^{44}{ }^{45}$ Individually, these tools are highly sensitive, however, a combined abnormal MRI and trajectory of abnormal GMA and HINE scores demonstrates the greatest diagnostic accuracy $(97.8 \%$ sensitivity and $99.2 \%$ specificity) at 3 months $\mathrm{CA}^{46}$ The GMA evaluates the quality of an infant's early spontaneous movement patterns, which reflects central nervous system integrity and function. ${ }^{47} 48$ An abnormal/ absent GMA at 3 months CA is highly predictive of CP in 'high-risk' infants, ${ }^{45}$ and may be a marker for other adverse NDOs. ${ }^{35} 4749-51$ Due to the time-sensitive nature of the GMA (at 11-17 weeks CA), the HINE is recommended to assess an infant's neurological development between 3 and 24 months CA. ${ }^{44}$ The HINE also provides an insight into CP topography (unilateral vs bilateral) ${ }^{52} 53$ and severity (ambulant vs non-ambulant, GMFCS I-III vs IV-V) ${ }^{54-58}$ While the GMA and HINE are relatively easy to administer, trained clinicians are required to evaluate and interpret scores.

In Australia, the rate of $\mathrm{CP}$ is estimated to be $50 \%$ higher for Indigenous children, ${ }^{8}$ with the rate of prenatally or perinatally acquired CP almost three times that of non-Indigenous infants. ${ }^{59}$ Indigenous infants with $\mathrm{CP}$ are more likely to be born extremely preterm ( $<28$ weeks) and LBW than non-Indigenous infants with CP, increasing their risk of functional severity. ${ }^{860}$ Indigenous infants are five times more likely to acquire CP PNN, which is associated with an increased severity of $\mathrm{CP}$ and linked to socioeconomic conditions. ${ }^{8230}$ In addition to higher rates of CP diagnosis, Indigenous children with CP have poorer cognitive and gross motor outcomes and a higher proportion of comorbidities, being twice as likely to have visual impairments and $50 \%$ have a codiagnosis of epilepsy. ${ }^{859}$ Accurate Australian data pertaining to the prevalence of $\mathrm{CP}$, age of diagnosis, rates of referral and access to early intervention in Indigenous infants remain unknown.

\section{Autism spectrum disorder}

Autism spectrum disorder (ASD) describes a group of heterogeneous NDDs characterised by core difficulties with social interaction and the presence of restrictive and repetitive patterns of interest or behaviours. ${ }^{61}$ Many individuals with ASD demonstrate associated impairments in cognition, challenging behaviours, communication and motor function. ${ }^{38} 62$ With a $42 \%$ increase in prevalence from 2015 to 2018 in Australia, ${ }^{63}$ the diagnosis of ASD continues to be commonly made after 2 years and frequently not until school age (ie, average 6years ${ }^{64}$ ), limiting timely early intervention. ${ }^{65}$

Early motor abnormalities, ${ }^{38} 3966-68$ reduced verbal skills, differences in social interactions ${ }^{69} 70$ and ASDrelated infant behaviours may be detected in children with ASD from 6 months CA; however, there are few ASD screening and diagnostic tools for infants $<12$ months of age. ${ }^{71}$ The Autism Observational Schedule in Infants (AOSI) evaluates the presence of ASD-related behaviours, in infants aged 6-18 months. ${ }^{71-74}$ Elevated AOSI scores at 12 and 18 months CA are associated with ASD diagnosis at 2 and 3 years of age, and are predictive of social-communication difficulties in high-risk infants at 2 years. $^{72-75}$ Atypical responses to specific test items, including eye contact, social interest and orienting to name are discriminative between high-risk infants with a subsequent diagnosis, high-risk infants without subsequent diagnosis and low risk infants. ${ }^{74}$ Differences in infant motor development ${ }^{6768} 77$ and the quality of early infant movements may provide additional insights into ASD-related outcomes. ${ }^{35474951}$ Studies investigating the use of GMA for prediction of ASD in high-risk infants identified that $>60 \%$ of children with a later confirmed diagnosis had abnormal or absent fidgety movements (FMs) at 12-16 weeks of age. ${ }^{3549} 51$ Universal screening tools such as the Ages and Stages Questionnaire $\left(\mathrm{ASQ}^{78}\right)$ and the Rapid Neurodevelopmental Assessment $\left(\mathrm{RNDA}^{79}\right)$ identify infants with atypical cognitive, social and communication development, but require further investigation regarding the predictive ability of ASDrelated behaviours.

There is a paucity of data relating to the prevalence of ASD in Australian Indigenous populations. ${ }^{80}$ While some studies have investigated the incidence of ASD and intellectual disability among specific Indigenous communities, accurate prevalence remains relatively unknown, with reported inconsistencies impacted by differences in cultural conceptualisation of disability, misdiagnosis and decreased awareness of ASD among Indigenous communities. $^{315} 6480-83$ There is growing concern that Indigenous children are misdiagnosed or missing out on an ASD diagnosis, ${ }^{62}$ supporting the need for culturally sensitive early diagnostic tools and services.

\section{Foetal alcohol spectrum disorder}

Alcohol exposure in utero can result in adverse outcomes across multiple neurodevelopmental domains including cognition, motor skills, brain structure, language, academic achievement, attention and adaptive behaviour. ${ }^{84-86}$ Foetal Alcohol Spectrum disorder (FASD) is the diagnostic term used for individuals who are exposed to alcohol prenatally and demonstrate severe impairment in three or more neurodevelopmental domains. ${ }^{85} 87$ Diagnosis according to the Australian Guide is categorised as either; FASD with three sentinel facial features or FASD with $<3$ sentinel facial features, indicating the presence 
or absence of facial dysmorphology specific to prenatal alcohol exposure (PAE) in the first trimester. ${ }^{8586}$ The coexistence of multiple comorbidities can complicate FASD diagnosis and further impact the long-term sequalae. ${ }^{88}$ FASD can be associated with an increased risk of physical health conditions, ${ }^{89}$ poor mental health, substance misuse and involvement in the criminal justice system. ${ }^{90}$ These lifelong consequences are extremely costly to the individual, family, health, education, disability and justice systems. ${ }^{91} 92$

The Australian Guide to the assessment and diagnosis of FASD $^{87}$ recommends early intervention, however early diagnosis and provision of appropriate treatment strategies are underdeveloped. ${ }^{93}$ In the absence of facial dysmorphology, there are few accurate early biomarkers for infants at risk of FASD. ${ }^{84} 878894$ Diagnostic assessments are complex, time consuming and require a multidisciplinary team of specialised clinicians. ${ }^{86}$ Furthermore, most of the recommended standardised neurodevelopmental assessments are for children $>2$ years. ${ }^{87}$ The use of standardised screening tools $<6$ months $\mathrm{CA}$, such as GMA and HINE may enable the accurate detection of neurodevelopmental delay, which could lead to earlier diagnosis of FASD.

The reported prevalence of FASD and patterns of PAE in Australia is variable, due to complexities with missed or misdiagnosis, practitioners not enquiring about prenatal alcohol use and availability of diagnostic services. ${ }^{93} 9596$ In Australia, rates of FASD in some Indigenous populations are among the highest globally, impacted by the interplay of biological and psychosocial risk factors. ${ }^{10697}$ In one remote community $19 \%$ of school-aged children had a FASD diagnosis, 25 times higher than the global rate. ${ }^{97}$ Furthermore, the prevalence of FASD $(47 \%)$ among Aboriginal young people (13-17 years) in custody in WA is almost six times higher than that of nonIndigenous adolescents in custody. ${ }^{96}$ The subsequent effect of PAE on developmental trajectory underpins the need for culturally sensitive, early screening tools to enable detection of infants who are high risk of FASD.

While there is emerging data on the prevalence and profile of adverse NDOs and NDDs in the Indigenous population, 81014152799 the focus has been on diagnosis of specific NDDs in early childhood. The aim of this cohort study is to investigate the use of early standardised screening tools (such as GMA and HINE) to determine risk status of infants aged $\leq 12$ months $\mathrm{CA}$, for a later diagnosis of CP, ASD, FASD and/or other substantial developmental delay in an 'at risk' Australian Indigenous birth cohort.

\section{OVERVIEW OF AIMS \\ Broad aim}

The primary aim of the current study is to investigate the impact of early screening for Indigenous infants at risk of adverse NDOs due to prenatal, birth and early life factors, in terms of:
1. Diagnostic accuracy, clinical utility and cultural appropriateness of early infant neurodevelopmental assessments to accurately predict a later 'at risk' diagnosis at 12 months CA.

2. Impact of perinatal variables, maternal factors and caregiver mental health on the developmental outcomes of Indigenous infants at risk of adverse NDOs in Queensland.

A comprehensive list of study aims and hypotheses are outlined in table 1 .

\section{METHODS}

\section{Study design}

This multisite prospective cohort study of 120 Indigenous infants will be conducted in Queensland, Australia, commencing in 2021 and will run for 2 years, with planned completion for 2023. The methodological design follows the Strengthening the Reporting of Observational Studies in Epidemiology guidelines. ${ }^{100}$

\section{Participants}

A cohort of 120 Indigenous infants with identified risk factors for adverse NDOs will be recruited. Recruitment will occur over an 18-month period (birth years 20202022) from the NICU, SCN, paediatric wards and outpatient clinics across Queensland.

\section{Inclusion criteria}

Infants eligible for screening will be those aged 0-9 months CA with one or both biological parents identifying as Aboriginal and/or Torres Strait Islander, who meet the following criteria:

1. Pregnancy complications, LBW $(<2500 \mathrm{~g})$, born preterm $(<37$ weeks gestation) or at term with Hypoxic Ischaemic Encephalopathy, 5 min Apgar <6, history of neurological risk factors (eg, admission to NICU/ SCN, congenital abnormalities, SGA, seizures), PNN complications (eg, head injury, stroke, infection, non-accidental injury), maternal risk factors that may impact neonatal outcomes (eg, medical conditions, antenatal substance use) or family history of adverse NDOs and/or sibling with a diagnosed NDD.

2. Reside in Queensland.

\section{Exclusion criteria}

Infants with major congenital or chromosomal abnormalities identified as part of routine medical care.

\section{Recruitment procedures}

Infants will be recruited through Queensland Hospital and Health services (HHS) and Aboriginal Community Controlled Health Organisations with ethics and governance approvals in place (see acknowledgments). The study will be introduced to parents or caregivers of infants who meet eligibility criteria by an Indigenous Liaison Officer (ILO) or member of staff from the recruiting sites. If families are interested in participating and consent to being contacted, a member of the research team will 
Table 1 LEAP-CP: early detection study aims and hypotheses

Aim 1: To determine the predictive accuracy, of the General Movements Assessment (GMA), the General Movements Motor Optimality Score (MOS), the Hammersmith Infant Neurological Examination (HINE), the Rapid Neurodevelopmental Assessment (RNDA) and the Ages and Stages-Aboriginal adaptation (ASQ-TRAK) to predict a later outcome at 12 months CA of 'at high risk' of (i) CP or (ii) Adverse Neurodevelopmental Outcome (non-CP) or (iii) typically developing in Indigenous infants.

$\mathrm{H} 1 \mathrm{a}$ Sensitivity to detect CP at 12 months CA in Indigenous infants will be $>98 \%$ for abnormal GMA (Absent Fidgety, Abnormal Fidgety) at 3 months CA and $>90 \%$ for suboptimal HINE score ( $<60$ and/or $>5$ asymmetries) at 6 months CA.

H1b Specificity to detect CP at 12 months CA in Indigenous infants will be $>90 \%$ for abnormal GMA (Absent Fidgety, Abnormal Fidgety) at 3 months CA and $>85 \%$ for suboptimal HINE score (<60 and/or $>5$ asymmetries) at 6 months CA.

$\mathrm{H1C}$ Indigenous infants with a confirmed or 'at risk' diagnosis of CP at 12 months will have a Motor Optimality Score (MOS) between 8 and 14 (GMFCS I-III) or <8 (GMFCS IV and V) at 3-5 months CA, infants with a diagnosis of 'at risk' of adverse NDOs (non-CP) at 12 months CA will have a MOS $<21$ at $3-5$ months CA.

H1d The sensitivity and specificity of the GMA and MOS to detect an adverse NDO (non-CP) at 12 months CA will be less than that of CP.

H1e Sensitivity and specificity to detect adverse NDOs (non-CP) at 12 months CA will be $\geq 81 \%$ and $>71 \%$, respectively, for suboptimal HINE score $(<65)$ at 6 months or $(<70)$ at 9 months CA.

$\mathrm{H} 1 \mathrm{f}$ Indigenous infants who score 'at risk' on $\geq 1$ domain the ASQ-TRAK at 6 months CA (domain-specific cut-offs gross motor $<23$, fine motor $<26$, communication $<30$, problem-solving $<28$, personal-social $<26$ ) will have a diagnosis of 'at risk' of adverse NDOs (non-CP) and/or CP at 12 months CA.

H1g Indigenous infants who score moderate to severe on any domain of the RNDA at 6 months CA will have good to excellent specificity (>0.8) compared with poor to fair sensitivity (0.6-0.8) to detect 'at risk' of CP and/or adverse NDOs (non-CP) at 12 months CA.

Aim 2: To determine the neurological (HINE), motor (PDMS-2), cognitive (BSID-III), developmental (PEDI-CAT/ASQ-TRAK) and behavioural (ITSEA) profiles of Indigenous infants with a diagnosis of 'at risk' of specific NDDs (i) CP, (ii) ASD, (iii) FASD and/or (iv) adverse NDO (non-specific) or (v) typically developing/borderline at 12 months CA compared with normative data.

$\mathrm{H} 2 \mathrm{a}$ Indigenous infants at high risk of CP at 12 months CA will score HINE<70 (GMFCS I-III), or $\leq 40$ (GMFCS IV-V); BSIDIII $>2$ SD below the mean ( $50 \%$ cognitive scale, $25 \%$ communication scale), PDMS-2 $>1$ SD below the mean (GMFCS I-III) or $>2$ SD below the mean (GMFCS IV-V) and PEDI-CAT >1SD below the mean (GMFCS I-III) or $>2$ SD below the mean (GMFCS IV-V) (mobility scale).

$\mathrm{H} 2 \mathrm{~b}$ Indigenous infants with ASD symptomology at 12 months CA will have a greater number of risk markers on the AOSI and/or will score HINE<70, on average score $>1$ SD below the mean on the BSID-III (communication scale, cognitive scale) and PDMS-2, PEDI-CAT >2SD below the mean (personal/social scale), ITSEA $\geq 1.5$ SD below the mean (competence domain) and/or>1.5SD above the mean (externalising, internalising and dysregulation domains).

$\mathrm{H} 2 \mathrm{C}$ Indigenous infants with FASD symptomology at 12 months CA will have microcephaly, $<3$ sentinel facial features and significant impairment ( $\geq 2$ SD below the mean or equivalent) on $\geq 3$ developmental domains including motor (PDMS-2 total motor quotient, PEDI-CAT mobility), neurological ( $<70$ on the HINE), cognitive (BSID-III cognitive subscale, PEDICAT daily activities), communication (BSID-III language composite score), adaptive behaviour/social skills (PEDI-CAT personal/social scales, ITSEA subdomains).

$\mathrm{H} 2 \mathrm{~d}$ Indigenous infants at risk of adverse NDOs (non-specific) at 12 months will have significant impairment (>2 SD below the mean) on one domain and/or or mild to moderate impairment ( $>1$ SD below mean) in $\geq 2$ domains including motor (PDMS-2 total motor quotient, PEDI-CAT mobility), neurological (<70 on the HINE), cognitive (BSID-III cognitive subscale, PEDI-CAT daily activities), communication (BSID-III language composite score), adaptive behaviour/social skills (PEDICAT personal/social scales, ITSEA).

$\mathrm{H} 2 \mathrm{e}$ Indigenous infants typically developing ( $\leq 1 \mathrm{SD}$ below the mean or equivalent on all developmental domains) or borderline (mild delay; between 1 and 2SD below the mean on one domain) at 12 months CA will score $>70$ on the HINE (neurological), and $\leq 1$ SD below the mean on the PDMS-2, BSID-III, PEDI-CAT and ITSEA (motor, cognition, communication, self-care and personal/social scales, behaviour).

Aim 3: To determine the clinimetric properties of outcome and/or predictive measures used to assess a cohort of 'at risk' Indigenous infants (GMA, HINE, RNDA, ASQ-TRAK, BSID-III, PDMS-2, PEDI-CAT and ITSEA) in terms of (i) construct validity, (ii) reliability, (iii) cultural acceptability and (iv) clinical utility/feasibility.

H3a Indigenous infants who are assessed to have $\geq 2$ neurodevelopmental impairments (NDI) and/or score moderate to severe impairment on any domain of the RNDA at 6 months and 12 months CA will have suboptimal HINE scores at 6 $(<65)$ and $12(<70)$ months CA.

$\mathrm{H} 3 \mathrm{~b}$ Indigenous infants who score 'at risk' on the communication $(<16)$ and/or problem-solving $(<28)$ domains of the ASQTRAK at 12 months CA will score $\geq 2$ SD below the mean on the language and/or cognitive domains of the BSID-III at 12 months CA. 


\section{Table 1 Continued}

H3c Indigenous infants who score 'at risk' on the gross motor (<22) and/or fine motor (<35) domains of the ASQ-TRAK at 12 months CA will score $\geq 2$ SD below the mean on the Gross Motor and/or Fine Motor Quotients of the PDMS-2 at 12 months CA.

H3d Indigenous infants who score 'at risk' on the personal-social (<22) domain of the ASQ-TRAK at 12 months CA will score $\geq 2$ SD below the mean on the corresponding domain of the PEDI-CAT and ITSEA at 12 months CA.

$\mathrm{H} 3 \mathrm{e}$ There will be strong inter-rater reliability and agreement $(k>0.8)$ between clinicians and community health workers for the HINE, RNDA and ASQ-TRAK.

H3f The clinical utility and cultural acceptability of screening tools used to predict later neurodevelopmental outcomes of Indigenous infants at $\leq 9$ months (GMA, HINE, RNDA and ASQ-TRAK) will be higher than that of tools used to measure developmental outcomes at 12 months CA (PDMS-2, BSID-III, PEDI-CAT and ITSEA).

Aim 4: To determine the relationship between (i) perinatal variables, (ii) maternal risk factors and outcomes of (i) motor, (ii) cognition and (iii) development for Indigenous infants at 12 months CA.

$\mathrm{H} 4 \mathrm{a}$ Adverse perinatal variables including, gestational age ( $<37$ weeks), low birth weight $(<2500 \mathrm{~g})$, events that signify complications during labour and delivery, adverse neonatal medical complications and PNN events including, infection, non-accidental injury, cerebrovascular accident, will be significantly associated with lower scores on neurological, motor, cognitive, developmental and behavioural assessments at 12 months CA (HINE, PDMS-2, BSID-III, ASQ-TRAK, PEDICAT, RNDA and ITSEA).

H4b Maternal risk factors (significant maternal medical conditions, antenatal medical complications and treatment, antenatal substance use and social risk factors as determined by the Social Risk Index), will be associated with lower scores on neurological, motor, cognitive, developmental and behavioural assessments at 12 months CA (HINE, PDMS-2, BSID-III, ASQ-TRAK, PEDI-CAT, RNDA and ITSEA).

$\mathrm{H} 4 \mathrm{C}$ Elevated caregiver stress, anxiety and depression on the DASS-21 will be associated with lower scores on neurological, motor, cognitive, developmental and behavioural measures in Indigenous infants at 12 months CA (HINE, PDMS-2, BSID-III, ASQ-TRAK PEDI-CAT, RNDA and ITSEA).

AOSI, Autism Observational Schedule in Infants; ASQ-TRAK, Ages and Stages Questionnaire-Aboriginal Adaptation; BSID, Bayley Scales of Infant Development; CA, corrected age; CP, cerebral palsy; FASD, Foetal Alcohol Spectrum disorder; GMA, General Movements Assessment; GMFCS, Gross Motor Functional Classification System; HINE, Hammersmith Infant Neurological Examination; ITSEA, Infant Toddler SocialEmotional Assessment; MOS, Motor Optimality Score; NDD, neurodevelopmental disorders; NDO, neurodevelopmental outcomes; PDMS, Peabody Developmental Motor Scales; PEDI-CAT, Paediatric Evaluation of Disability Inventory-Computer Adaptive Test; RNDA, Rapid Neurodevelopmental Assessment .

contact the family and provide information regarding the study, including a culturally adapted parent information statement. The research team member, who is not associated with the infant's care, will explain the study in more detail and answer all parent questions prior to seeking informed consent for study participation. Families will be given the option to verbally discuss the parent information sheet with an ILO or Indigenous Community Health Worker (CHW) prior to providing written informed consent to participate. Once signed consent is obtained, the infant will be enrolled in the study and will commence the relevant screening assessments.

\section{Sample size}

This study aims to predict a later diagnosis of (i) typical development or 'at risk' of specific NDD, (ii) CP, (iii) ASD, (iv) FASD and/or (v) adverse NDO (non-specific) in a population of Indigenous infants with known exposure to early life risk factors. The projected sample size of 120 Indigenous infants is based on the expected number of new diagnoses of CP, ASD, FASD or adverse NDOs over an 18-month period at the study sites. The Cairns and Townsville hospitals have a potential combined total of 1400 infants admitted to their NICU and SCN's per year. Approximately $38 \%(n=540)$ of these infants have one or both biological parents who identify as Indigenous. The proportion of participating children with an adverse NDO we are likely to observe in the LEAP-CP cohort has been estimated by combining data from Australian data registers with data from a retrospective audit of a cohort of high-risk infants admitted to the Townsville Hospital NICU or SCN during 2019-2020.

The Western Australia Cerebral Palsy register is the register that has reported rates of CP in Indigenous children for the longest duration and has a current estimate of $4.01 \mathrm{CP}$ cases per 1000 births. Incidence of ASD in Indigenous Australian children is hypothesised to approximately equal non-Indigenous rates, at between 7 and 15 ASD cases per 1000 births. ${ }^{6} 80$ Incidence of FASD in Indigenous Australians is estimated at 17 FASD cases per $1000,{ }^{101}$ but could be as much as 10-times higher in some remote communities. ${ }^{97}$ The overall number of Indigenous children who have either developmental delay or an adverse NDO may range from $10 \%$ in low risk cohorts ${ }^{14}$ to $30 \%$ in high-risk remote communities. ${ }^{10}$

A retrospective audit of high-risk Indigenous children admitted to the Townsville Hospital neonatal unit or SCN identified 16 children with known outcomes at 12-24 months CA. Of these children, 25\% were at high 
risk of CP, $25 \%$ were at risk of a non-CP NDO, $31 \%$ had a non-neuromotor delay, while $19 \%$ had no neurodevelopmental concerns. Overall, $>80 \%$ of these children were classed as having at least mild delay, although it should be noted that these children were at higher risk for an NDO than those who will participate in the LEAP-CP cohort. For the 120 children recruited to the LEAP-CP cohort, we estimate approximately one-third (33\%) will be identified as being at risk of an NDO. This will allow us to estimate the diagnostic accuracy of tools to within $\pm 12 \%$ (sensitivity) and $\pm 9 \%$ (specificity), assuming accuracy of $80 \%$. When identifying characteristics associated with an NDO, assuming we have a binary predictor variable with equal numbers in each category and a baseline risk of 0.33 , we will have $80 \%$ power (alpha $=0.05$ ) to identify relative risks of 1.75 or greater.

\section{Patient and public involvement}

Members of Indigenous communities at each participating site across Queensland have and will continue to be actively engaged at all stages of study development and the research programme. Key community stakeholders including community elders, Aboriginal and Torres Strait Islander health workers, Indigenous researchers and people with lived experience as parents of infants/children with cerebral palsy, have been involved in all steps of study design. Consultation and input particularly guided the cultural adaptation and development of culturally safe and sensitive delivery, presentation and feedback of information to families and caregivers including early screening, recruitment and consent processes and key measures to be utilised throughout the programme. Consultation and engagement with key stakeholders will continue to be sought throughout programme delivery, final analysis and data interpretation.

Strategies targeting key components of cultural safety and sensitivity, consultation and codesign, capacity building and sustainability, are fundamental to the cultural framework that underpins this study and will be led by Indigenous coinvestigators. Consumer engagement will be embedded into the study at key screening and outcome timepoints to evaluate parent/caregiver and CHW experience and satisfaction with the screening process and appropriateness and feasibility of assessments.

The final results of the study will be presented in collaborative workshops involving key stakeholders, Aboriginal and Torres Strait Islander community members and personnel at each participating site at the conclusion of the study. Information on the study results will also be reported to all participants as summary data presented to each participating family.

\section{Data collection methods}

Data collection will commence following consent and enrolment. Extensive perinatal data will be collected from the infant's medical records, including gestational age, birth weight, sex, birth history, neonatal course and maternal risk factors (see online supplemental S1:
LEAP-CP Medical Checklist: Part 1-Perinatal data and birth history). Primary caregivers will complete a baseline parent questionnaire that collects detailed sociodemographic information including, maternal and paternal education and employment, social support, family structure and prenatal exposures (see online supplemental S1: LEAP-CP Medical Checklist: Part 2-Socio-demographic Information). Caregivers will be given the option to complete this form either independently or during a supported interview with an ILO or Indigenous CHW.

Participants will be screened at two time points, (1) birth to 5 months CA, and (2) 4-9 months CA. Infants can enter the study at any time between birth and 9 months $\mathrm{CA}$, and will commence the relevant screening protocol based on their age at study entry. Outcome measures will be completed at 12 months CA (figure 1).

\section{Birth to 5 months CA (screening stage 1)}

Infants recruited prior to 9 weeks CA will be assessed as an inpatient or outpatient using the General Movements Assessment (GMA, writhing period). ${ }^{48}$ The assessment will be recorded by a member of staff who is trained in the procedural guidelines for GMA and uploaded to a secure server. Between 12 and 17 weeks, CA infants will be assessed twice using the GMA (fidgety period) via video taken at a clinic appointment or by an application on the caregiver's phone and later uploaded to a secure server. The General Movements smartphone application (Baby Moves $^{102}$ ) will be set up on the caregiver's phone by a member of hospital staff or the research team on recruitment to the study. Culturally adapted written/pictorial instructions will be provided to guide caregivers how to video their infant's movements, with support offered by an ILO/CHW. A reminder will be sent via the Baby Moves app to caregivers to ensure videos are recorded at two time-points (ideally at 12 and 14 weeks CA). All GMA videos will be viewed and scored by a minimum of two assessors who are advanced trained by the General Movement Trust and are masked to the participant's identity and medical history. The General Movements Motor Optimality Score (MOS) will be assessed and scored simultaneously using the infant's fidgety GMA videos by the same independent assessors. ${ }^{103}$

\section{Assessments at 4-9 months CA (screening stage 2)}

The second stage of screening will occur from 4 to 9 months CA. Infants will attend an appointment with a local healthcare worker where they will be assessed using the HINE, RNDA, Ages and Stages-Aboriginal Adaptation (ASQ-TRAK) and clinical assessment of physical features of FASD (photograph with or without direct measurement). The mother or primary caregiver will complete the Depression Anxiety Stress Scale (DASS-21). Developmental assessments will be administered and scored live by a trained allied health professional, paediatrician, CHW or child health nurse and will be video recorded to allow for independent scoring by a masked assessor. Results from all early screening assessments will be 


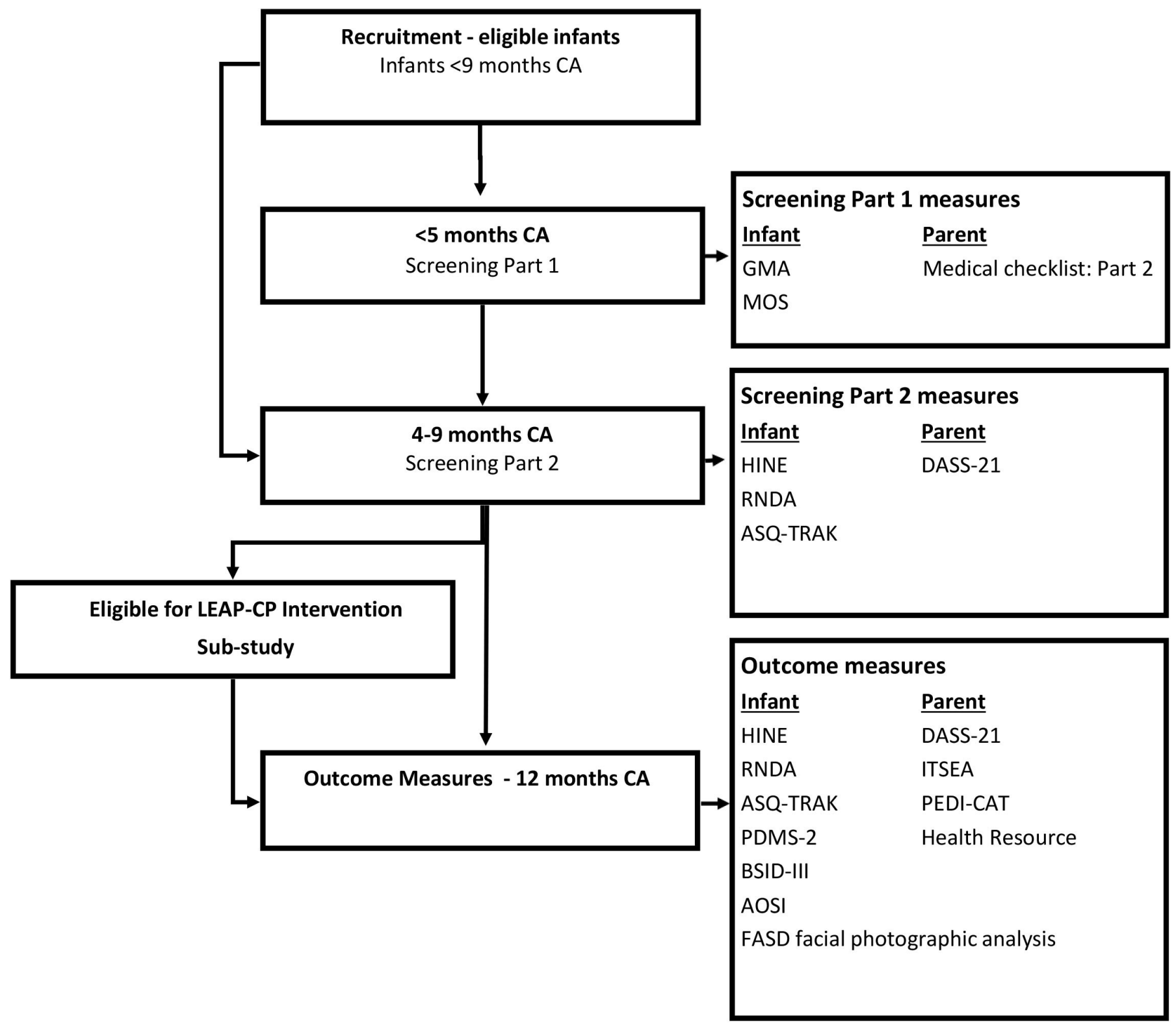

Figure 1 LEAP-CP prospective cohort study timeline. AOSI, Autism Observation Schedule in Infants; ASQ-TRAK, Ages and Stages Questionnaire-Aboriginal Adaptation; BSID-III, Bayley Scales of Infant Development third Edition; DASS-21, Depression Anxiety Stress Scale; FASD, facial photographic analysis; GMA, Prechtl's General Movements Assessment; HINE, Hammersmith Infant Neurological Examination; ITSEA, Infant Toddler Social Emotional Assessment; MOS, General Movements Assessment Motor Optimality Score; PEDI-CAT, Paediatric Evaluation of Disability Inventory-Computer Adaptive Test; PMDS-2, Peabody Developmental Motor Scales second edition; RNDA, Rapid Neurodevelopmental Assessment.

provided to the infant's treating team with parental/caregiver consent. Infants who are rated absent or abnormal FMs on the GMA at 3 months CA and/or receive a suboptimal HINE score at 4-9 months CA are considered to be at 'high risk' of CP and/or adverse NDO and will be referred to the LEAP-CP intervention trial and linked with local community health services.

\section{Outcomes at 12 months CA}

At 12 months CA ( \pm 1 month), all participants will attend an appointment at their local health service. Infants will be assessed by a trained allied health clinician on the HINE, RNDA, ASQ-TRAK, Peabody Developmental
Motor Scales- second edition (PDMS-2) and the cognitive and language scales of the Bayley Scales of Infant Development- third edition (BSID-III). Infants will complete diagnostic specific outcome measures (1) Autism Observation Scale for Infants (AOSI; ASD) and (2) clinical assessment of physical features of FASD (photograph with or without direct measurement) to determine the presence of symptomology and risk of a later diagnosis of ASD and/or FASD. Assessments will be recorded to allow independent scoring by an assessor masked to the infant's risk of adverse NDOs, medical history and previous assessment findings. A paediatrician, masked 
to the infant's developmental history, will complete the medical assessment for differential diagnosis from video and photographic (FASD symptomology) assessment (see online supplemental S2: LEAP CP: 12-month Medical Assessment-Differential Diagnosis). Caregivers will complete the DASS-21, Infant Toddler Social-Emotional Assessment (ITSEA), Paediatric Evaluation of Disability Inventory-Computer Adaptive Test (PEDI-CAT) and health resource and information questionnaire, either independently or as an interview supported by an ILO or CHW (see online supplemental S3: LEAP-CP Medical and Allied Health Resource Form). Child outcomes will be provided to parents/caregivers via written report and results will be forwarded to the infant's treating team with parental/caregiver consent.

\section{MEASURES}

\section{Infant predictor variables}

Prechtl's Qualitative Assessment of General Movements

Prechtl's Qualitative Assessment of General Movements (GMA) is a predictive and discriminative tool used to longitudinally observe the quality of early spontaneous movement patterns in infants from birth to 20 weeks CA. The GMA demonstrates high diagnostic accuracy, 97\% specific and 95-98\% sensitive, at 3 months CA for detecting infants with a later diagnosis of CP. ${ }^{44-46}$ General movements (GMs) are assessed over specific time periods as either writhing (birth-9 weeks CA) or fidgety (9-20 weeks CA). Writhing movements are rated as normal, characterised by complex, variable, fluent movements involving the whole body, or abnormal, classified as either poor repertoire, cramped synchronised or chaotic. ${ }^{47} 48$ FMs) re present from 9 weeks until voluntary, more purposeful movements become predominant. ${ }^{48}$ Typical (normal) FMs are defined as small amplitude, multidirectional movements, of the trunk, neck and limbs, of moderate speed, that are continuous in the awake infant, except during periods of crying, fussing and focused attention. ${ }^{47}$ Atypical FMs are classified as either absent or abnormal, referring to either the absence (absent) or exaggeration (abnormal) of typical FMs. ${ }^{47}$ While the absence of FMs at 3 months is the best predictor of $\mathrm{CP}^{45}$ abnormal GMA at writhing age has been associated with later cognitive delays, ${ }^{104}$ and abnormal fidgety GMA (abnormal or absent) has been associated with early motor delay related to prenatal substance use, ${ }^{36}$ and is emerging as a potential marker of atypical movement patterns in infants later diagnosed with ASD. ${ }^{35105}$ Assessment of the GMA requires a $3-5$ min video of the infant lying in supine, during periods of active wakefulness, free from distractions. In this study, writhing GMA will be completed only if infants are recruited between birth and 4 weeks post-term age. Fidgety GMA will occur at two timepoints (ideally between 12 and 17 weeks CA) to give optimal opportunity for FMs to emerge within the 'peak' window ${ }^{106}$ and will be scored by at least two advanced trained assessors, masked to the infant's medical and clinical history, to decrease the potential impact of measurement bias.

\section{General Movements Motor Optimality Score}

The MOS is a more detailed analysis of an infant's fidgety GMA to determine their concurrent motor repertoire at 3-5 months CA by observing postural patterns and movement quality, across five subcategories. ${ }^{103}$ The score of each subcategory; quality of FMs, quality of movement patterns, age-adequate movement repertoire, postural patterns and movement character, combines to give a total MOS ranging from 5 to $28 .{ }^{103}$ Scores $>25$ are optimal and indicative of typical outcomes, scores ranging from 20 to 24 are mildly reduced and $\mathrm{MOS}<20$ requires intervention. ${ }^{57103}$ The presence of specific movement patterns and low scores on the MOS are predictive of a later CP diagnosis and may provide early markers for CP severity, subtype and topography. ${ }^{103} 107108$ Increasing evidence supports the MOS as a prognostic indicator for adverse NDOs (non-CP), and therefore, its function as a transdiagnostic screening tool. Suboptimal MOS scores have been associated with later outcomes of minor neurological dysfunction, language impairments, learning and behavioural difficulties in children without a CP diagnosis. ${ }^{109110}$ Additionally, a monotonous movement character was identified in almost $60 \%$ of infants who were prenatally exposed to alcohol and addictive substances, ${ }^{36}$ has been found in infants with later diagnoses of NDDs (non-CP) including $\mathrm{ASD}^{51}$ and genetic disorders, ${ }^{103}$ and, has been linked to cognitive delays at school age in a cohort of high-risk infants. ${ }^{111}$ The MOS will be assessed and scored concurrently with fidgety GMA, by the same masked, advanced trained assessors.

\section{Hammersmith Infant Neurological Evaluation}

The HINE is a quantifiable, neurological examination for infants aged 2-24 months CA. ${ }^{112}$ It is predictive of suboptimal NDOs with $90 \%$ accuracy in predicting CP in infants aged $>18$ weeks CA. ${ }^{4113}$ The HINE is divided into three sections, section 1 consists of 26 items that assesses infant neurological function across five domains: cranial nerves, posture, tone, reflexes and movements. Sections 2 and 3 evaluate the infant's motor development and state of behaviour, these sections are not scored. ${ }^{112}$ Each item from section 1 is scored from zero to three, where a score of three is indicative of an optimal item response. Item scores are combined to determine a global optimality score, with a maximum possible score of 78. An infant's global score is compared with age specific optimality scores and cut-offs to determine risk of adverse NDOs. ${ }^{112}$ Suboptimal HINE scores $(<65,<70)$ at 6 and 9-12 months, respectively, are associated with significant delays and/or CP at 2 years,${ }^{37}$ with further age specific cutpoints $(<57,<60,<63$ and $<66)$ at $3,6,9$ and 12 months, respectively, predictive of a later diagnosis of $\mathrm{CP}^{54}$ Infants with hemiplegic $\mathrm{CP}$ or milder neurological disorders may score above age-specific cut offs. ${ }^{52}{ }^{54}$ Differences observed in item responses between the left and right 
sides are recorded as asymmetries and are combined to obtain a total asymmetry score. A total of $\geq 5$ asymmetries are associated with increased risk of unilateral CP. ${ }^{52}$ The HINE is accessible, quick to administer, approximately 5-10 min and has good interobserver reliability, even when performed by less experienced staff. ${ }^{112}$

\section{Rapid Neurodevelopmental Assessment}

The RNDA is a criterion-based instrument, originally designed to comprehensively assess and identify children 'at risk' of neurodevelopmental impairment (NDI) living in low to middle income countries with limited access to health screening services. ${ }^{79}$ The screening tool is intended for use by lay health workers and has been successfully integrated into Aboriginal Health clinics at Gidgee Healing in Mt Isa, Queensland. ${ }^{114} 115$ The instrument assesses the functional status of children aged 0-9 years to determine the presence and severity of NDIs across multiple domains. ${ }^{79116117}$ Infants aged 1-24 months $\mathrm{CA}$ are assessed across eight domains: gross motor, fine motor, vision, hearing, speech, cognition, behaviour and seizures. Each item is scored on a 4-point scale, as normal $=0$, mild $=0.5$, moderate $=1$ or severe $=2$ impairment. The sum of item scores are used to determine the presence and degree of impairment for each domain. ${ }^{118}$ The RNDA has been validated in infants $<2$ years CA to determine the presence of NDI versus no NDI ${ }^{79}$ and demonstrates moderate to high agreement with the Bayley Scales of Infant Development-second edition and BSID-III for identifying infants aged $<12$ months CA with and without NDIs. ${ }^{79} 119$ The RNDA has good face validity, evident in its acceptability by caregivers, clinicians and infants and has been culturally adapted for use in other countries. ${ }^{79} 119$ The RNDA has high inter-rater reliability among medical professionals across the domains of gross motor $(\mathrm{k}=1.00)$, behaviour $(\mathrm{k}=1.00)$, fine motor $(\mathrm{k}=0.93)$ and seizures $(\mathrm{k}=0.91)$, with moderate agreement for cognition $(\mathrm{k}=0.80)$, hearing $(\mathrm{k}=0.78)$ and speech $(0.63) .{ }^{79} \mathrm{~A}$ similar level of agreement was also demonstrated between local community workers and trained health professionals across cognition, speech, behaviour, gross and fine motor domains. ${ }^{119}$ Administration time for the RNDA is between 30 and $45 \mathrm{~min}$ and must be completed by a trained clinician or health worker. ${ }^{79}$

Ages and Stages Questionnaire, Australian Aboriginal Adaptation The ASQ-TRAK (adapted from the Ages and Stages Questionnaire third edition ${ }^{78}$ ) is the only developmental screening tool that has been adapted and validated specifically for use in an Australian Indigenous context. ${ }^{120121}$ The ASQ-TRAK demonstrates acceptable accuracy, sensitivity $(71 \%)$, specificity $(92 \%)$, for detecting developmental concerns in Indigenous children, and, has demonstrated concurrent validity with the BSID-III, with moderate correlation between corresponding domain scores on both tools. ${ }^{120}$ The ASQ-TRAK consists of interview-based questionnaires available for children aged 2, 6, 12, 18, 24, 36 and 48 months, assessing outcomes across five areas: communication, gross motor, fine motor, problem-solving and personal-social. ${ }^{122}$ The screening tool contains the same items and scoring as the ASQ-3 but is based on a caregiver interview, with opportunity for the child to demonstrate skills. Culturally relevant adaptations to the ASQ-3 include, translation into local language and item modifications to ensure cultural relevance. ${ }^{122}$ Individual items are assessed as 'yes', 'sometimes' or 'not yet' to ascertain a score of 10,5 or 0 , respectively. Individual, domainspecific, item scores are combined to determine the total domain score (maximum=60). Scores are compared with domain specific cut-offs to determine risk of developmental delay, with further assessment recommended for infants who score below the cut-off, or 'at risk', for any domain. ${ }^{121}$ The ASQ-TRAK has proven face validity and was determined to be culturally relevant and acceptable by Aboriginal healthcare workers and parents. ${ }^{122} 123$ The screener takes 30-60 min to complete and can be administered by trained healthcare workers. ${ }^{120}$

\section{Infant Outcome measures}

Outcomes will be assessed at 12 months CA ( \pm 2 weeks) by a trained allied health clinician and videoed for scoring by a researcher masked to perinatal data and earlier assessment data points.

\section{Peabody Developmental Motor Scales second edition}

Infant primary motor outcomes at 12 months CA will be assessed using the PDMS-2, a standardised, normreferenced measure used to evaluate the gross and fine motor development of children aged birth to 6 years. ${ }^{124}$ The gross motor component is comprised of four subtests: reflexes, stationary, locomotion and object manipulation. Two subtests, grasping and visual-motor integration, form the fine motor component. ${ }^{124}$ Individual items are allocated a score from zero to two based on performance, 0 (unable to perform), 1 (partial performance) or 2 (correct performance). Subtest raw scores are used to determine motor outcomes and ascertain the presence and severity of motor delay. The PDMS-2 has demonstrated predictive validity, sensitivity $(92 \%)$, to identify abnormal development at 18 months in preterm infants assessed at 8 months. ${ }^{125}$ The assessment has concurrent validity with both the BSID-III ${ }^{126}$ and the Gross Motor Functional Measure. ${ }^{127}$ The PDMS-2 is responsive to change in a population of infants ${ }^{128}$ and toddlers with CP. ${ }^{129}$ The assessment takes $45-60$ min to complete, with formal training not required for the administration and scoring of the PDMS-2.

Bayley Scales of Infant Development-third edition (BSID-III)

The BSID-III is the gold standard, norm-referenced assessment for measuring the development of infants and toddlers, aged 1-42 months, to determine infant cognitive and communication outcomes at 12 months CA. The BSID-III comprises five scales: cognitive, language, motor, social-emotional and adaptive behaviour. Items are administered in a standardised procedure and scored as either 
credit $=1$ or no credit $=0$. A composite score of $>2$ SD below the mean on any scale is indicative of delay and supports the need for intervention. ${ }^{130}$ In this study, we will use the BSID-III cognitive and language scales to assess infant outcomes at 12 months CA. The BSID-III (cognitive and language scales) have demonstrated predictive validity for outcomes on the Weschler Preschool and Primary Scale of Intelligence-III at 4 years of age. ${ }^{131}$ Internal consistency reliability and test retest reliability were determined for the composite and subtest scores on the Bayley III cognitive and language scales across all ages, with higher reliability demonstrated in age groups $>6$ months of age. ${ }^{130}$ The BSID-III low motor/low vision version will be used to improve validity when assessing children with mild-tomoderate motor and/or vision impairment. ${ }^{132}$ While the Bayley IV is now available, ${ }^{133}$ the Bayley III will be used in this study to compare this Indigenous cohort to other non-Indigenous Australian cohorts. ${ }^{134}$ A trained professional is required to administer the assessment, average time taken to complete varies with age and ranges from approximately 50-90 min. ${ }^{130} 135$

\section{The Paediatric Evaluation of Disability Inventory-Computer Adaptive Test}

Developmental outcomes in self-care, mobility and social function will be assessed at 12 months CA using the PEDI-CAT, a standardised, norm-referenced assessment of independence in self-care. ${ }^{136}$ The PEDI-CAT has been designed for use from birth to 21 years of age and has been Rasch analysed in children with disability and typical development. ${ }^{136}$ The instrument measures functional outcomes across four domains, daily activities, the ability to perform living skills, mobility, the ability to move around the home and in the community, and, social/cognitive the ability to participate and effectively engage in social situations. Responsibility, the fourth domain, will not be assessed in this study. ${ }^{136}$ The tool is administered via a web-based application (Q-global), allowing parents / caregivers to self-report their child's independence on each domain. The PEDI-CAT uses an item bank which automatically lowers the number of test items dependent on how the child is scoring. ${ }^{136} 137$ Items are scored on a 4-point difficulty scale with responses ranging from unable to easy. Normative scores are reported as a $\mathrm{T}$-score and an age percentile range (<5th, 5th-25th). The PEDI-CAT has good discriminant validity in CP populations, between children with and without disability, and, demonstrates concurrent validity with the Wee-FIM in children with brain injury and developmental disabilities. ${ }^{138-140}$ The PEDI-CAT is frequently used as an assessment to determine entry and allocation of resources for children entering the Australian National Disability Insurance Scheme (NDIS) ${ }^{141}$ The test is valid, reliable and responsive in this population, takes $10-15$ min to complete, and test administration requires no formal training. ${ }^{140} 142$

\section{Infant Toddler Social and Emotional Assessment}

The ITSEA is a 168 item, parent-report questionnaire designed to evaluate social-emotional and behavioural competencies and difficulties in infants aged 12 months to 3 years old. ${ }^{143}$ The instrument measures items across four behavioural domains: externalising, internalising, dysregulation and competencies. Items are scored on a 3-point (0-2) scale, not true/rarely (0), somewhat true/sometimes (1), and, very true often (2). ${ }^{144}$ The ITESA is discriminative between high and low risk infants with social-emotional difficulties at 12 months of age, ${ }^{144}$ and demonstrates strong test-retest reliability $(\alpha=0.75-0.91){ }^{145}$

\section{Diagnostic assessments}

At 12 months, CA infants will be assessed by a paediatrician who will complete a medical assessment for differential diagnosis (online supplemental S2: LEAP-CP 12-month Medical Assessment) including documenting the presence of ASD and FASD symptomology. Functional severity, motor type and distribution of $\mathrm{CP}$ will be ascertained for infants who have a confirmed or high-risk diagnosis of CP.

\section{Diagnosis of cerebral palsy}

Confirmed or high-risk CP will be diagnosed according to published guidelines, ${ }^{146-148}$ based on clinical history (LEAP-CP Medical checklist) and videoed HINE and PDMS-2 assessments.

\section{Motor type and distribution}

Motor type will be classified as spastic, dystonic, ataxic, choreoathetosis, mixed CP or unclassifiable according to Surveillance of Cerebral Palsy in Europe guidelines. ${ }^{147}$ Motor distribution will be classified by number of limbs impaired and unilateral or bilateral distribution by an independent assessor.

\section{Functional severity}

The Gross Motor Functional Classification System (GMFCS) has validity, reliability and stability for the classification and prediction of motor function of children with CP aged 2-12 years. ${ }^{149-151}$ The GMFCS extended and revised version, $0-2$ year descriptors, will be used to classify the gross motor abilities of infants at 12 months CA. ${ }^{152}$ The GMFCS has been correlated with CP motor type and distribution. ${ }^{153}$

The Mini Manual Abilities Classification Scale (MACS) is used to classify hand function and abilities in children aged 0-4 years and is the gold standard for classifying infant's ability to handle objects in daily activities. ${ }^{154} \mathrm{An}$ independent assessor will use videos to observe and classify children in one of five functional categories for each scale.

\section{ASD symptomology}

The Autism Observation Scale for Infants (AOSI) will be used to measure ASD symptomology at 12 months CA. ${ }^{155}$ The AOSI, a semistructured observational tool, was 
designed to assess the presence and emergence of specific ASD-related behaviours in infants aged 6-18 months. ${ }^{71} 155$ The experimenter led tool assesses 18 items, individual item scores range from 0 to 3 and are combined to obtain a total score, with higher scores indicating elevated risk of ASD behaviours. ${ }^{71}$ The presence of seven or more risk markers at 12 months was $52 \%$ sensitive and $74 \%$ specific for an ASD diagnosis at 3 years. ${ }^{75}$ The AOSI differentiates between high-risk and low-risk infants at 12-18 months. ${ }^{73} 7576156$ Inter-rater reliability for individual items and total scores is excellent (0.92 and 0.93, respectively) at 12 months and test-retest reliability is acceptable. ${ }^{71}$

\section{FASD symptomology}

\section{Assessment of PAE}

The Alcohol Use Disorders Identification TestConsumption (AUDIT-C) will be used to ascertain the potential level of fetal risk associated with maternal alcohol use during pregnancy (prepregnancy and postpregnancy recognition). The validated, sex-specific version of the instrument comprises three questions as a standardised method of assessing maternal alcohol consumption. ${ }^{157} 158$ An AUDIT-C score of $\geq 5$ or a reported consumption of 5 or more standard drinks on one occasion is associated with increased risk of FASD. ${ }^{87} 158$

\section{Sentinel facial features}

Clinical assessment of facial features will be completed via direct measurement (where possible) and/or assessed from a photograph, analysed using the University of Washington facial analysis software. ${ }^{159}$ Smooth philtrum and thin upper lip will be assessed using the University of Washington Caucasian or African American (depending on what is individually appropriate) lip-philtrum guide ( 1 or 2), where a rank of 4 or 5 meets criteria for FASD sentinel facial features. The Scandinavian (Stromland) chart will be utilised to measure palpebral fissure length where a result of $\geq 2 \mathrm{SD}$ below the mean $(<3$ rd percentile) is significant. ${ }^{87} 160$ Standard frontal and oblique facial photographs will be analysed using the FAS Facial Photographic Analysis Software for facial dysmorphology assessment. $^{159}$

\section{Severe neurodevelopmental impairment}

Assessment of impairment will target five of the 10 neurodevelopmental domains that reflect known areas of brain function affected by PAE. ${ }^{87}$ Infant's neurological, motor, cognitive, language and adaptive and social skills will be assessed using standardised outcome measures at 12 months CA. Severe impairment will be defined as score of $\geq 2$ SD below the mean, or equivalent, on the HINE (neurological), PDMS-2 (motor), Bayley III (cognitive and language scales), PEDI-CAT (adaptive/social) and ITSEA (behaviour) ${ }^{87}$ Infants with a head circumference less than <3rd centile and/or abnormal brain imaging including structural brain abnormalities will also be considered as criteria for severe brain structure/neurological impairment. ${ }^{87}$ Presence and severity of impairment will be determined by assessors blinded to the infant's clinical history and predictor assessment outcomes.

\section{Special considerations for infants}

In children under 6 years of age with all three sentinel facial features and microcephaly, a diagnosis of FASD with three Sentinel Facial Features can be made, regardless of confirmed PAE and in the absence of severe neurodevelopment impairment in three domains. In the absence of microcephaly, children under 6 years of age with all three sentinel facial features are considered 'at risk of FASD', whether PAE is confirmed or unknown. ${ }^{87}$

\section{Parent/caregiver Outcome measure}

Depression Anxiety Stress Scale (DASS-21)

Parent or primary caregiver mental health status will be assessed at two time-points (screening stage 2 and infant 12 month outcomes) using the DASS-21, a 21-item, selfreported tool designed to measure the presence of the negative emotional states of depression, anxiety and stress. ${ }^{161}$ Individual items assess the presence of symptoms across three subscales (depression, anxiety and stress). Participants use a 4-point scale to reflect and rate the extent to which they have experienced each symptom over the past week. Item scores are combined to determine the severity; normal, mild, moderate, severe or extremely severe, for each emotional state. ${ }^{161}$ The DASS-21 has demonstrated concurrent validity with the Beck depression and anxiety inventories ${ }^{162} 163$ and has been utilised in a population of Indigenous mothers to assess maternal emotional well-being. ${ }^{164}$

\section{Covariates and descriptive measures}

Perinatal data

An extensive record of antenatal, birth history and the neonatal course will be collected at the time of infant enrolment from medical records (see online supplemental S1: LEAP-CP Medical checklist). Data collected will include the following:

1. Demographic data including gestational age, birth weight, sex and multiple birth status.

2. Perinatal events that signify complications during labour and delivery, indicating increased risk of adverse NDO.

3. Neonatal medical complications associated with adverse NDOs including early brain injury, infection, necrotising enterocolitis, respiratory distress, bronchopulmonary dysplasia, postnatal infant steroid therapy, neonatal surgery, retinopathy of prematurity, prolonged use of oxygen and feeding status at discharge.

4. Maternal risk factors that may impact neonatal outcomes, including, antenatal medical complications and treatment, medical conditions (diabetes mellitus and epilepsy), antenatal substance use, mental health status and family history of adverse NDOs.

\section{Clinical neuroimaging}

Cranial ultrasound (CUS) and MRI assessment findings will be collected and retrieved from Hospital records. 
Abnormal MRI, including white matter injury, cortical and grey matter lesions and brain maldevelopments may be indicative of neuroanatomy abnormalities predictive of adverse NDOs. ${ }^{45}$ MRI findings will be utilised in the diagnostic process for CP and symptomology of FASD.

\section{Demographic data}

Demographic data will be collected at two time points:

The LEAP-CP Medical Checklist: Part 2 (online supplemental S1), completed at study enrolment, details information regarding family structure and supports, primary language spoken at home, maternal and paternal education and employment status. The Social Risk Index (SRI) and the AUDIT-C questionnaire will be embedded into this document to ascertain level of family social risk and infant PAE. ${ }^{158} 165$

The LEAP-CP Medical Resource form (online supplemental S3), completed at or prior to the 12-month CA appointment, to provide information regarding their child's development, access to services and eligibility and/or access to NDIS funding.

\section{Social Risk Index}

The 12-point SRI measures six aspects of social status; family structure, language spoken at home, maternal age at birth and primary caregiver education, occupation and income. Risk items are scored from 0 to 2, with a lower score associated with lower risk. Overall family risk scores will be classified as lower $(\leq 1)$ or higher social risk $(>2) .{ }^{166167}$

\section{DATA MANAGEMENT AND ANALYSIS PLAN}

All data will be entered into a REDcap database by ID number (reidentifiable). Data analysis will be carried out using Stata V.16.0 ${ }^{168}$ statistical software package. Predictor and outcome variables will be identified as continuous, categorical or binary. Analysis will explore means, variability and distributions of continuous variables and the rate of occurrence and distribution of binary variables. Infants will be categorised at 12 months $\mathrm{CA}$ as at risk of specific NDD, (1) CP, (2) ASD, (3) FASD (as defined by the presence of disorder specific symptomology) and/or (4) adverse NDO (non-specific, defined as $>2$ SD below the mean or equivalent on one developmental domain and/ or $>1$ SD below mean in $\geq 2$ domains) or (v) typically developing $(\leq 1 \mathrm{SD}$ below the mean or equivalent on all developmental domains) or borderline (mild delay; between 1 and 2SD below the mean on one domain). Logistic regression analysis (binary outcomes), linear regression (continuous outcomes) and multinomial logistic regression (categorical outcomes) will be used to determine any associations between predictor and outcome variables. Diagnostic statistics, including sensitivity, specificity, positive and negative predictive values and accuracy of the predictive assessments (GMA, MOS, HINE, RNDA and ASQ-TRAK) will be determined with 95\% CIs based on an outcome of 'at risk' of specific NDD,
(1) CP, (2) ASD, (3) FASD and/or (4) adverse NDO (nonspecific) at 12 months CA. Perinatal variables, social and environmental data, caregiver mental health outcomes (DASS-21) and clinical neuroimaging will be utilised as descriptive measures and covariates in regression models.

\section{DISCUSSION}

Results of this study will inform service delivery of follow-up pathways for Indigenous infants at risk of adverse NDOs and their families. Our findings will inform culturally sensitive practice and enable clinicians to select both clinically meaningful and culturally appropriate tools to identify Indigenous infants at high risk of adverse NDOs at an earlier age. Early detection will fast track families to access early intervention services for Indigenous infants and families and enable early referral to the targeted motor and cognitive training in the LEAP-CP clinical trial and or mainstream allied health services to promote optimal outcomes.

Infants will be recruited early to establish discharge pathways and a follow-up plan, with local services. Engagement, and established connections with local health services will enable locally trained Indigenous CHWs to assist in the screening process for infants and families living remotely, with support provided via telehealth as required. Culturally adapted resources, developed in partnership with Indigenous co-investigators and consumers, will be utilised to facilitate safe and sensitive communication and practices throughout the screening and diagnostic process for infants and families. This study aims to foster local Indigenous workforce capacity through skill development and training opportunities and build on current models of care to enable feasible and sustainable early detection programmes for 'at risk' Indigenous infants. Assisting existing services to implement culturally appropriate screening programmes will ensure these strategies and pathways can be embedded into regular service delivery models at the conclusion of the study.

\section{Ethics and dissemination of findings}

Ethics committee approvals were obtained from the appropriate Indigenous ethics/governance committees (see acknowledgements). There are no known health or safety risks associated with participation in any aspect of the described study. Cultural adaptations will be made to all resources and throughout the study families will be given the option to verbally discuss any questions or concerns with an ILO or CHW to ensure comprehension of concepts, cultural and language barriers are addressed. Families can withdraw their child from the study at any time without explanation, without any penalty from staff at the treating or referring hospital or health service, or any effect on their child's care. Data collected in this study will be securely stored in a coded reidentifiable form (by 
ID number at the University of QLD). Summary data of outcome measures will be shared with the treating clinician and/or team with the parent/caregiver's permission.

Findings of this study will be of interest to medical, allied health and community health workers, working with Indigenous infants and families in urban, rural and remote communities.

Findings will be disseminated via peer-reviewed publications, conference presentations, clinical practice guidelines outlining culturally appropriate screening tools and sensitively communicating a diagnosis and resources including culturally adapted factsheets.

\section{Author affiliations}

${ }^{1}$ Queensland Cerebral Palsy and Rehabilitation Research Centre, Child Health Research Centre, The University of Queensland, Brisbane, Queensland, Australia

${ }^{2}$ Townsville Hospital and Health Service, Townsville, Queensland, Australia

${ }^{3}$ Menzies Health Institute Queensland, Griffith University, Nathan, Queensland, Australia

${ }^{4}$ Child Health Research Centre, The University of Queensland, Brisbane, Queensland, Australia

${ }^{5}$ Department of Neonatology, University Medical Centre Groningen, Groningen, The Netherlands

Acknowledgements The LEAP-CP programme received ethical approval from the Far North Queensland Health Research Ethics Committee (HREC/2019/QCH/50533 (Sep ver 2)-1370), the Townsville HHS Human Research Ethics Committee (HREC/ QTHS/56008), the University of Queensland Medical Research Ethics Committee (2020000185/HREC/2019/QCH/50533) and the Children's Health Queensland HHS Human Research Ethics Committee (HREC/20/QCHQ/63906) and research governance approval from the Apunipima Cape York Health Council Research Governance Committee (03/2019) and Gurriny Yealamucka Health Services Aboriginal Corporation (12/2020). We acknowledge the key contributions of the Aboriginal and Torres Strait Islander peoples who have engaged, provided consultation and codesign to the cultural framework and study design and have shared knowledge to ensure the cultural sensitivity and safety of the programme, to reflect key concepts of being, knowing and doing. In particular, key community stakeholders from Gurriny Yealamucka health service, Apunipima Cape York Health council and community members with lived experience who have shared their stories and expertise. We acknowledge the support of the Queensland Cerebral Palsy and Rehabilitation Research Centre clinical research team (Ms Anya Gordon, Ms Lucy Fogarty, Ms Donna Fallon, Ms Chloe Taifalos, Ms Ellena Oakes, Ms Sarah Gibson, Ms Bernadette Shannon, Ms Kym Morris and Ms Christine Finn) for their clinical inputs and role as advanced General Movements assessors.

Collaborators Collaborators who have provided assistance with patient care and data collection: Gurriny Yealamucka Health Service Aboriginal Corporation, Apunipima Cape York Health Council, Townsville HHS, Cairns and Hinterland HHS, Children's Health Queensland HHS.

Contributors CRL had substantial input into study design, ethics applications and was the lead author for the protocol manuscript. She will take a lead role in the recruitment, data collection and analysis of the study. KB is part of CRL's advisory team and is the chief investigator for the LEAP RCT. She had substantial input into all components of the study design including methods and key outcome measures, ethics applications and engagement with key community leaders and provided feedback and revision throughout manuscript development. LM-R is leading the cultural framework for the study including adaptations and modifications to study resources. She lead the cultural engagement and co-design with key community stakeholders for the study and provided feedback and revision throughout manuscript development, particularly with regard to the cultural sensitivity and safety of the study. RSW lead the statistical design and analysis component of the paper and contributed to the study design in particular sample size and statistical analysis. He provided feedback and revision throughout manuscript development. NR contributed in particular to the FASD diagnostic and symptomology design and component of the manuscript. She also provided key insight to working with First Nations communities. She provided feedback and revision throughout manuscript development. AFB provided expert consultation regarding the use of the general movements assessment in particular the more detailed analysis using the motor optimality score. He provided key additions to the manuscript and study design and will continue to work as a consultant for the study. He provided feedback and revision throughout manuscript development. MB is part of CRL's advisory team, she provided key input into the study design and methodology in the context of regional Queensland. Her contributions regarding rehabilitation medicine were integral to the manuscript development. She provided feedback and revision throughout manuscript development. RNB is CRL's primary advisor and provided substantial input into all components of the study design. She provided high level feedback and revision throughout the manuscript development and signed off on final revisions. RNB led the engagement with LM-R with First Nations communities and was integral to all parts of the study including ethics applications.

Funding The LEAP-CP detection study is supported by project grants awarded by the Research Foundation, Cerebral Palsy Alliance; PHD02619 (CRL), PG05318 (KB), the National Health and Medical Research Council; Investigator grant 1195602 (RNB), Australia Early Career Fellowship 1145212 (KB), NHMRC-EU Horizon Grant 11941280 (KB/RNB), Perpetual IMPACT Philanthropy grant IPAP2020/0808 (RNB), Children's Health QLD 50276 (KB) and The University of Queensland PhD RTP scholarship (CRL).

Competing interests None declared.

Patient consent for publication Not required.

Provenance and peer review Not commissioned; externally peer reviewed.

Supplemental material This content has been supplied by the author(s). It has not been vetted by BMJ Publishing Group Limited (BMJ) and may not have been peer-reviewed. Any opinions or recommendations discussed are solely those of the author(s) and are not endorsed by BMJ. BMJ disclaims all liability and responsibility arising from any reliance placed on the content. Where the content includes any translated material, BMJ does not warrant the accuracy and reliability of the translations (including but not limited to local regulations, clinical guidelines, terminology, drug names and drug dosages), and is not responsible for any error and/or omissions arising from translation and adaptation or otherwise.

Open access This is an open access article distributed in accordance with the Creative Commons Attribution Non Commercial (CC BY-NC 4.0) license, which permits others to distribute, remix, adapt, build upon this work non-commercially, and license their derivative works on different terms, provided the original work is properly cited, appropriate credit is given, any changes made indicated, and the use is non-commercial. See: http://creativecommons.org/licenses/by-nc/4.0/.

\section{ORCID iDs}

Carly R Luke http://orcid.org/0000-0002-2732-1942

Robert S Ware http://orcid.org/0000-0002-6129-6736

\section{REFERENCES}

1 Menzies K. Understanding the Australian Aboriginal experience of collective, historical and intergenerational trauma. Int Soc Work 2019;62:1522-34.

2 Griffiths K, Coleman C, Lee V, et al. How colonisation determines social justice and Indigenous health - a review of the literature. $J$ Popul Res 2016;33:9-30.

3 Gilroy J, Donelly M, Colmar S. Twelve factors that can influence the participation of Aboriginal people in disability services. Australian Indigenous Health Bulletin 2016;16 http://healthbulletin.org.au/ articles/twelve-factors-that-can-influence-the-participation-ofaboriginal-people-in-disability-services

4 Australian Institute of Health and Welfare. The health and welfare of Australia's Aboriginal and Torres Strait Islander peoples. Canberra: AlHW, 2015.

5 Australian Bureau of Statistics. Aboriginal and Torres Strait Islander people with disability. Canberra: ABS, 2017.

6 Lilley R, Sedgwick M, Pellicano E. "We look after our own mob": aboriginal and torres strait Islander experiences of Autism. Sydney, Australia: Macquarie University, 2019.

7 DiGiacomo M, Delaney P, Abbott P, et al. 'Doing the hard yards': carer and provider focus group perspectives of accessing Aboriginal childhood disability services. BMC Health Serv Res 2013;13:326.

8 Blair E, Watson L, O'Kearney E, et al. Comparing risks of cerebral palsy in births between Australian Indigenous and non-Indigenous mothers. Dev Med Child Neurol 2016;58:36-42. 
9 Ou L, Chen J, Hillman K, et al. The comparison of health status and health services utilisation between Indigenous and non-Indigenous infants in Australia. Aust N Z J Public Health 2010;34:50-6.

10 Fitzpatrick JP, Latimer J, Olson HC, et al. Prevalence and profile of neurodevelopment and fetal alcohol spectrum disorder (FASD) amongst Australian Aboriginal children living in remote communities. Res Dev Disabil 2017;65:114-26.

11 DiGiacomo M, Davidson PM, Abbott P, et al. Childhood disability in Aboriginal and Torres Strait Islander peoples: a literature review. Int $J$ Equity Health 2013;12:7.

12 Bishop D, Pine D, Scott S. Rutter's child and adolescent psychiatry. John Wiley \& Sons, 2009.

13 Accardo J, Shapiro BK. Neurodevelopmental disabilities: beyond the diagnosis. Semin Pediatr Neurol 2005;12:242-9.

14 McDonald J, Webster V, Knight J, et al. The Gudaga study: development in 3-year-old urban Aboriginal children. J Paediatr Child Health 2014;50:100-6.

15 Bourke J, de Klerk N, Smith T, et al. Population-based prevalence of intellectual disability and autism spectrum disorders in Western Australia: a comparison with previous estimates. Medicine 2016;95:e3737.

16 Australian Institute of Health and Welfare. Australia's mothers and babies 2018: in brief. Perinatal statistics series no.36. Cat. no. PER 108. Canberra: AlHW, 2020.

17 Commonwealth of Australia Department of the Prime Minister and Cabinet. Closing the gap report, 2020.

18 Van Hus J, Potharst ES, Jeukens-Visser M. Motor impairments in very preterm-born children: links with other developmental deficits at 5 years of age. Develop Med \& Child Neurol 2013.

19 de Kieviet JF, Piek JP, Aarnoudse-Moens CS, et al. Motor development in very preterm and very low-birth-weight children from birth to adolescence: a meta-analysis. JAMA 2009;302:2235-42.

20 Saigal S, Doyle LW. An overview of mortality and sequelae of preterm birth from infancy to adulthood. Lancet 2008;371:261-9.

21 Doyle LW, Victorian Infant Collaborative Study Group. Evaluation of neonatal intensive care for extremely low birth weight infants in Victoria over two decades: II. efficiency. Pediatrics 2004;113:510-4.

22 Williams J, Lee KJ, Anderson PJ. Prevalence of motor-skill impairment in preterm children who do not develop cerebral palsy: a systematic review. Dev Med Child Neurol 2010;52:232-7.

23 Milner KM, Neal EFG, Roberts G, et al. Long-term neurodevelopmental outcome in high-risk newborns in resourcelimited settings: a systematic review of the literature. Paediatr Int Child Health 2015;35:227-42.

24 Gibberd AJ, Simpson JM, Jones J, et al. A large proportion of poor birth outcomes among Aboriginal Western Australians are attributable to smoking, alcohol and substance misuse, and assault. BMC Preg Child 2019;19:110.

25 Comino E, Knight J, Webster V, et al. Risk and protective factors for pregnancy outcomes for urban Aboriginal and non-Aboriginal mothers and infants: the Gudaga cohort. Matern Child Health $J$ 2012;16:569-78.

26 Kildea S, Gao Y, Hickey S, et al. Reducing preterm birth amongst Aboriginal and Torres Strait Islander babies: a prospective cohort study, Brisbane, Australia. EClinicalMedicine 2019;12:43-51.

27 Westrupp EM, D'Esposito F, Freemantle J, et al. Health outcomes for Australian Aboriginal and Torres Strait Islander children born preterm, low birthweight or small for gestational age: a nationwide cohort study. PLoS One 2019;14:e0212130.

28 Mclnerney C, Ibiebele I, Ford JB, et al. Benefits of not smoking during pregnancy for Australian Aboriginal and Torres Strait Islander women and their babies: a retrospective cohort study using linked data. BMJ Open 2019;9:e032763.

29 Homer CSE, Foureur MJ, Allende T, et al. 'It's more than just having a baby' women's experiences of a maternity service for Australian Aboriginal and Torres Strait Islander families. Midwifery 2012;28:E449-55.

30 D'Aprano AL, Carapetis JR, Andrews R. Trial of a developmental screening tool in remote Australian Aboriginal communities: a cautionary tale. J Paediatr Child Health 2011;47:12-17.

31 Nishimura T, Takei N, Tsuchiya KJ, et al. Identification of neurodevelopmental trajectories in infancy and of risk factors affecting deviant development: a longitudinal birth cohort study. Int $J$ Epidemiol 2016;45:543-53.

32 Talbott MR, Miller MR. Future directions for infant identification and intervention for autism spectrum disorder from a Transdiagnostic perspective. J Clin Child Adolesc Psychol 2020;49:688-700.

33 Johnson $\mathrm{MH}$, Gliga T, Jones E, et al. Annual research review: Infant development, autism, and ADHD-early pathways to emerging disorders. J Child Psychol Psychiatry 2015;56:228-47.
34 Finlay-Jones A, Varcin K, Leonard H, et al. Very Early Identification and Intervention for Infants at Risk of Neurodevelopmental Disorders: A Transdiagnostic Approach. Child Dev Perspect 2019;13:97-103.

35 Einspieler C, Sigafoos J, Bölte S, et al. Highlighting the first 5 months of life: general movements in infants later diagnosed with autism spectrum disorder or Rett syndrome. Res Autism Spectr Disord 2014;8:286-91.

36 Fjørtoft T, Brandal M, Brubakk AM, et al. Maternal alcohol and drug use during pregnancy affects the motor behaviour and general movements of infants aged 3-4 months. Early Hum Dev 2020;151:105171.

37 Romeo DM, Cowan FM, Haataja L, et al. Hammersmith infant neurological examination for infants born preterm: predicting outcomes other than cerebral palsy. Dev Med Child Neurol 2021;63:939-46.

38 Hedgecock JB, Dannemiller LA, Shui AM, et al. Associations of gross motor delay, behavior, and quality of life in young children with autism spectrum disorder. Phys Ther 2018;98:251-9.

39 Lim YH, Licari M, Spittle AJ, et al. Early motor function of children with autism spectrum disorder: a systematic review. Pediatrics 2021;147:e2020011270.

40 Report of the Australian cerebral palsy register: birth years 199520122018.

41 Rosenbaum P, Paneth N, Leviton A, et al. A report: the definition and classification of cerebral palsy April 2006. Dev Med Child Neurol Suppl 2007;109:8-14.

42 Novak I, Morgan C, Fahey M, et al. State of the evidence traffic lights 2019: systematic review of interventions for preventing and treating children with cerebral palsy. Curr Neurol Neurosci Rep 2020;20:3.

43 Galea C, Mcintyre S, Smithers-Sheedy H, et al. Cerebral palsy trends in Australia (1995-2009): a population-based observational study. Dev Med Child Neurol 2019;61:186-93.

44 Novak I, Morgan C, Adde L, et al. Early, accurate diagnosis and early intervention in cerebral palsy: advances in diagnosis and treatment. JAMA Pediatr 2017;171:897-907.

45 Bosanquet M, Copeland L, Ware R, et al. A systematic review of tests to predict cerebral palsy in young children. Dev Med Child Neurol 2013;55:418-26.

46 Morgan C, Romeo DM, Chorna O. The pooled diagnostic accuracy of neuroimaging, general movements, and neurological examination for diagnosing cerebral palsy early in high-risk infants: a case control study. J Clin Med 20191879;8:1. doi:10.3390/jcm8111879

47 Einspieler C, Prechtl H, Bos A. Prechtl's Method on the Qualitative Assessment of General Movements in Preterm Term and Young Infants: Cambridge University Press, Mac Keith Press. Clin Develop Med 2004.

48 Prechtl HF, Einspieler C, Cioni G, et al. An early marker for neurological deficits after perinatal brain lesions. Lancet 1997;349:1361-3.

49 Hadders-Algra M, Bouwstra H, Groen SE. Quality of general movements and psychiatric morbidity at 9 to 12 years. Early Hum Dev 2009;85:1-6.

50 Peyton C, Einspieler C. General movements: a behavioral biomarker of later motor and cognitive dysfunction in NICU graduates. Pediatr Ann 2018;47:e159-64.

51 Zappella M, Einspieler C, Bartl-Pokorny KD, et al. What do home videos tell us about early motor and socio-communicative behaviours in children with autistic features during the second year of life--An exploratory study. Early Hum Dev 2015;91:569-75.

52 Hay K, Nelin M, Carey H, et al. Hammersmith infant neurological examination asymmetry score distinguishes hemiplegic cerebral palsy from typical development. Pediatr Neurol 2018;87:70-4.

53 Romeo DMM, Guzzetta A, Scoto M, et al. Early neurologic assessment in preterm-infants: integration of traditional neurologic examination and observation of general movements. Eur J Paediatr Neurol 2008;12:183-9.

54 Romeo DMM, Cioni M, Palermo F, et al. Neurological assessment in infants discharged from a neonatal intensive care unit. Eur J Paediatr Neurol 2013;17:192-8.

55 Ricci D, Cowan F, Pane M, et al. Neurological examination at 6 to 9 months in infants with cystic periventricular leukomalacia. Neuropediatrics 2006;37:247-52.

56 Frisone MF, Mercuri E, Laroche S, et al. Prognostic value of the neurologic optimality score at 9 and 18 months in preterm infants born before 31 weeks' gestation. J Pediatr 2002;140:57-60.

57 Einspieler C, Utsch F, Brasil P, et al. Association of infants exposed to prenatal Zika virus infection with their clinical, neurologic, and developmental status evaluated via the general movement assessment tool. JAMA Netw Open 2019;2:e187235. 
58 Zang F-F, Yang H, Han Q, et al. Very low birth weight infants in China: the predictive value of the motor repertoire at 3 to 5 months for the motor performance at 12 months. Early Hum Dev 2016;100:27-32.

59 Martin T, Mclntyre S, Badawi N. A birds-eye view of the landscape of cerebral palsy for Aboriginal and Torres Strait Islander children in Australia. Dev Med Child Neurol 2020;62:38.

60 Forbes-Coe A, Jones A, Crowe M, et al. Perinatal factors that contribute to the prevalence of cerebral palsy in Townsville, North Queensland. J Neonatal Nurs 2018;24:208-12.

61 American Psychiatric Association. Diagnostic and statistical manual of mental disorders. 5th edn. Washington, DC, 2013.

62 Armstrong E, Jokel A. Language outcomes for preverbal toddlers with autism 2012;4.

63 Australian Bureau of Statistics. Autism in Australia, 2015. Australia, 2017.

64 Bent CA, Dissanayake C, Barbaro J. Mapping the diagnosis of autism spectrum disorders in children aged under 7 years in Australia, 2010-2012. Med J Aust 2015;202:317-20.

65 May T, Brignell A, Williams K. Autism spectrum disorder prevalence in children aged 12-13years from the longitudinal study of Australian children. Autism Res 2020;13:821-7.

66 Flanagan JE, Landa R, Bhat A, et al. Head lag in infants at risk for autism: a preliminary study. Am J Occup Ther 2012;66:577-85.

67 Esposito G, Venuti P, Maestro S, et al. An exploration of symmetry in early autism spectrum disorders: analysis of lying. Brain Dev 2009;31:131-8.

68 Teitelbaum P, Teitelbaum O, Nye J, et al. Movement analysis in infancy may be useful for early diagnosis of autism. Proc Natl Acad Sci U S A 1998;95:13982-7.

69 Franchini M, Duku E, Armstrong V, et al. Variability in verbal and nonverbal communication in infants at risk for autism spectrum disorder: predictors and outcomes. J Autism Dev Disord 2018;48:3417-31.

70 Barbaro J, Dissanayake C. Early markers of autism spectrum disorders in infants and toddlers prospectively identified in the social attention and communication study. Autism 2013;17:64-86.

71 Bryson SE, Zwaigenbaum L, McDermott C, et al. The autism observation scale for infants: scale development and reliability data. $J$ Autism Dev Disord 2008;38:731-8.

72 Bedford R, Gliga T, Shephard E, et al. Neurocognitive and observational markers: prediction of autism spectrum disorder from infancy to mid-childhood. Mol Autism 2017;8:49.

73 Gammer I, Bedford R, Elsabbagh M, et al. Behavioural markers for autism in infancy: scores on the autism observational scale for infants in a prospective study of at-risk siblings. Infant Behav Dev 2015;38:107-15.

74 Zwaigenbaum L, Bryson S, Rogers T, et al. Behavioral manifestations of autism in the first year of life. Int $J$ Dev Neurosci 2005;23:143-52.

75 Zwaigenbaum L, Bryson SE, Brian J, et al. Assessment of Autism symptoms from 6 to 18 months of age using the Autism observation scale for infants in a prospective high-risk cohort. Child Dev 2021;92:1187-98.

76 Brian J, Bryson SE, Garon N, et al. Clinical assessment of autism in high-risk 18-month-olds. Autism 2008;12:433-56.

77 Teitelbaum O, Benton T, Shah PK, et al. Eshkol-Wachman movement notation in diagnosis: the early detection of Asperger's syndrome. Proc Natl Acad Sci U S A 2004;101:11909-14.

78 Squires J, Bricker D. Ages and stages questionnaires, third edition (ASQ-3): a parent-completed child monitoring system. Baltimore: Paul H. Brookes Publishing Co., Inc, 2009.

79 Khan NZ, Muslima H, Begum D, et al. Validation of rapid neurodevelopmental assessment instrument for under-two-year-old children in Bangladesh. Pediatrics 2010;125:e755-62.

80 Bailey B, Arciuli J. Indigenous Australians with autism: a scoping review. Autism 2020;24:1031-46.

81 Roy M, Balaratnasingam S. Missed diagnosis of autism in an Australian Indigenous psychiatric population. Australas Psychiatry 2010;18:534-7.

82 Leonard H, Glasson E, Nassar N, et al. Autism and intellectual disability are differentially related to sociodemographic background at birth. PLoS One 2011;6:e17875.

83 Lilley R, Sedgwick M, Pellicano E. Inclusion, acceptance, shame and isolation: attitudes to autism in Aboriginal and Torres Strait Islander communities in Australia. Autism 2020;24:1860-73.

84 Lange S, Rovet J, Rehm J, et al. Neurodevelopmental profile of fetal alcohol spectrum disorder: a systematic review. BMC Psychol 2017;5:22.

85 Sokol RJ, Delaney-Black V, Nordstrom B. Fetal alcohol spectrum disorder. JAMA 2003;290:2996-9.
86 Cook JL, Green CR, Lilley CM, et al. Fetal alcohol spectrum disorder: a guideline for diagnosis across the lifespan. CMAJ 2016;188:191-7.

87 Bower C, Elliott E, on behalf of the steering group. Report to the Australian government department of health: Australian guide to the diagnosis of fetal alcohol spectrum disorder (FASD) 2016.

88 Popova S, Lange S, Shield K, et al. Comorbidity of fetal alcohol spectrum disorder: a systematic review and meta-analysis. Lancet 2016;387:978-87.

89 Reid N, Hayes N, Young SB. Caregiver-reported physical health status of children and young people with fetal alcohol spectrum disorder. J Develop Origin Health Dis 2020:1-8.

90 Streissguth AP, Bookstein FL, Barr HM, et al. Risk factors for adverse life outcomes in fetal alcohol syndrome and fetal alcohol effects. J Dev Behav Pediatr 2004;25:228-38.

91 Popova S, Lange S, Burd L, et al. The economic burden of fetal alcohol spectrum disorder in Canada in 2013. Alcohol Alcohol 2016;51:367-75

92 Popova S, Stade B, Bekmuradov D, et al. What do we know about the economic impact of fetal alcohol spectrum disorder? A systematic literature review. Alcohol Alcohol 2011;46:490-7.

93 Reid N. Fetal alcohol spectrum disorder in Australia: what is the current state of affairs? Drug Alcohol Rev 2018;37:827-30.

94 Wynn A, Rotheram-Borus MJ, Davis E, et al. Identifying fetal alcohol spectrum disorder among South African children at aged 1 and 5 years. Drug Alcohol Depend 2020;217:108266.

95 Reid N, Shelton D, Warner J, et al. Profile of children diagnosed with a fetal alcohol spectrum disorder: a retrospective chart review. Drug Alcohol Rev 2017;36:677-81.

96 Bower C, Watkins RE, Mutch RC, et al. Fetal alcohol spectrum disorder and youth justice: a prevalence study among young people sentenced to detention in Western Australia. BMJ Open 2018;8:e019605.

97 Fitzpatrick JP, Latimer J, Carter M, et al. Prevalence of fetal alcohol syndrome in a population-based sample of children living in remote Australia: the Lililwan project. J Paediatr Child Health 2015;51:450-7

98 Lange S, Probst C, Gmel G, et al. Global prevalence of fetal alcohol spectrum disorder among children and youth: a systematic review and meta-analysis. JAMA Pediatr 2017;171:948-56.

99 Williamson A, Gibberd A, Hanly MJ, et al. Social and emotional developmental vulnerability at age five in Aboriginal and nonAboriginal children in New South Wales: a population data linkage study. Int J Equity Health 2019;18:120.

100 von Elm E, Altman DG, Egger M, et al. The strengthening the reporting of observational studies in epidemiology (STROBE) statement: guidelines for reporting observational studies. The Lancet 2007;370:1453-7.

101 Popova S, Lange S, Shield K, et al. Prevalence of fetal alcohol spectrum disorder among special subpopulations: a systematic review and meta-analysis. Addiction 2019;114:1150-72.

102 Spittle AJ, Olsen J, Kwong A, et al. The baby moves prospective cohort study protocol: using a smartphone application with the general movements assessment to predict neurodevelopmental outcomes at age 2 years for extremely preterm or extremely low birthweight infants. BMJ Open 2016;6:e013446.

103 Einspieler C, Bos AF, Krieber-Tomantschger M, et al. Cerebral palsy: early markers of clinical phenotype and functional outcome. J Clin Med 2019;8:1616.

104 Olsen JE, Cheong JLY, Eeles AL, et al. Early general movements are associated with developmental outcomes at $4.5-5$ years. Early Hum Dev 2020;148:105115.

105 Phagava H, Muratori F, Einspieler C, et al. General movements in infants with autism spectrum disorders. Georgian Med News 2008;156:100-5 https://europepmc.org/article/med/18403821

106 Kwong AKL, Olsen JE, Eeles AL, et al. Occurrence of and temporal trends in fidgety General movements in infants born extremely preterm/extremely low birthweight and term-born controls. Early Hum Dev 2019;135:11-15.

107 Bruggink JLM, Cioni G, Einspieler C, et al. Early motor repertoire is related to level of self-mobility in children with cerebral palsy at school age. Dev Med Child Neurol 2009;51:878-85.

108 Yang H, Einspieler C, Shi W, et al. Cerebral palsy in children: movements and postures during early infancy, dependent on preterm vs. full term birth. Early Hum Dev 2012;88:837-43.

109 Bruggink JLM, Einspieler C, Butcher PR, et al. Quantitative aspects of the early motor repertoire in preterm infants: do they predict minor neurological dysfunction at school age? Early Hum Dev 2009;85:25-36.

110 Butcher PR, van Braeckel K, Bouma A, et al. The quality of preterm infants' spontaneous movements: an early indicator of intelligence 
and behaviour at school age. $J$ Child Psychol and Psychiatry 2009;50:920-30.

111 Fjørtoft T, Grunewaldt KH, Løhaugen GCC, et al. Assessment of motor behaviour in high-risk-infants at 3 months predicts motor and cognitive outcomes in 10 years old children. Early Hum Dev 2013;89:787-93.

112 Haataja L, Mercuri E, Regev R, et al. Optimality score for the neurologic examination of the infant at 12 and 18 months of age. $J$ Pediatr 1999;135:153-61.

113 Romeo DM, Ricci D, Brogna C, et al. Use of the Hammersmith infant neurological examination in infants with cerebral palsy: a critical review of the literature. Dev Med Child Neurol 2016;58:240-5.

114 Shanley DC, Hawkins E, Page M, et al. Protocol for the Yapatjarrathati project: a mixed-method implementation trial of a tiered assessment process for identifying fetal alcohol spectrum disorders in a remote Australian community. BMC Health Serv Res 2019;19:649.

115 Reid N, Hawkins E, Liu W, et al. Yarning about fetal alcohol spectrum disorder: outcomes of a community-based workshop. Res Dev Disabil 2021;108:103810.

116 Khan NZ, Muslima H, Shilpi AB, et al. Validation of rapid neurodevelopmental assessment for 2- to 5-year-old children in Bangladesh. Pediatrics 2013;131:e486-94.

117 Khan NZ, Muslima H, El Arifeen S, et al. Validation of a rapid neurodevelopmental assessment tool for 5 to 9 year-old children in Bangladesh. J Pediatr 2014:164:1165-70.

118 Khan NZ, Muslima H. Rapid neurodevelopmental assessment of children, a practical guide for health professionals. Dhaka, Bangladesh: Bangladesh Protibondhi Foundation, 2012.

119 Thompson L, Peñaloza RA, Stormfields K, et al. Validation and adaptation of rapid neurodevelopmental assessment instrument for infants in Guatemala. Child Care Health Dev 2015;41:1131-9.

120 Simpson S, D'Aprano A, Tayler C, et al. Validation of a culturally adapted developmental screening tool for Australian Aboriginal children: early findings and next steps. Early Hum Dev 2016;103:91-5.

121 D'Aprano A. Ages and stages questionnaires Third Edition - guide to using the ASQ-TRAK. 3rd edn. Paul H. Brookes Publishing Co, 2017.

122 D'Aprano A, Silburn S, Johnston V, et al. Adaptation of the ages and stages questionnaire for remote Aboriginal Australia. Qual Health Res 2016;26:613-25.

123 D'Aprano A, Silburn S, Johnston V, et al. Culturally appropriate training for remote Australian Aboriginal health workers: evaluation of an early child development training intervention. J Dev Behav Pediatr 2015;36:503-11.

124 Folio M, Fewell R. Peabody developmental motor scales: examiner's manual. 2nd edn. Austin, Tex: PRO-ED, Inc, 2000.

125 Darrah J, Piper M, Watt MJ. Assessment of gross motor skills of atrisk infants: predictive validity of the Alberta infant motor scale. Dev Med Child Neurol 1998:40:485-91.

126 Connolly BH, McClune NO, Gatlin R. Concurrent validity of the Bayley-III and the Peabody developmental motor Scale-2. Pediatr Phys Ther 2012;24:345-52.

127 Kolobe TH, Palisano RJ, Stratford PW. Comparison of two outcome measures for infants with cerebral palsy and infants with motor delays. Phys Ther 1998;78:1062-72.

128 Palisano RJ, Kolobe TH, Haley SM, et al. Validity of the Peabody developmental gross motor scale as an evaluative measure of infants receiving physical therapy. Phys Ther 1995;75:939-48.

129 Wang H-H, Liao H-F, Hsieh C-L. Reliability, sensitivity to change, and responsiveness of the peabody developmental motor scales-second edition for children with cerebral palsy. Phys Ther 2006;86:1351-9.

130 Bayley N. Bayley scales of infant and toddler development. PsychCorp, Pearson, 2006.

131 Bode MM, D'Eugenio DB, Mettelman BB, et al. Predictive validity of the Bayley, third edition at 2 years for intelligence quotient at 4 years in preterm infants. J Dev Behav Pediatr 2014;35:570-5.

132 Visser L, Ruiter SAJ, Van der Meulen BF, et al. Validity and suitability of the Bayley-III low motor/vision version: a comparative study among young children with and without motor and/or visual impairments. Res Dev Disabil 2013;34:3736-45.

133 Bayley N, Aylward G. Bayley scales of infant and toddler development. 4th edn. San Antonio: Harcourt, 2019.

134 Elliott C, Alexander C, Salt A, et al. Early moves: a protocol for a population-based prospective cohort study to establish General movements as an early biomarker of cognitive impairment in infants. BMJ Open 2021;11:e041695.
135 Spittle AJ, Doyle LW, Boyd RN. A systematic review of the clinimetric properties of neuromotor assessments for preterm infants during the first year of life. Dev Med Child Neurol 2008:50:254-66.

136 Haley SM, Coster WJ, Dumas HM, et al. Accuracy and precision of the pediatric evaluation of disability inventory computer-adaptive tests (PEDI-CAT). Dev Med Child Neurol 2011;53:1100-6.

137 Dumas H, Fragala-Pinkham M, Haley S, et al. Item bank development for a revised pediatric evaluation of disability inventory (PEDI). Phys Occup Ther Pediatr 2010;30:168-84.

138 Shore BJ, Allar BG, Miller PE, et al. Evaluating the discriminant validity of the pediatric evaluation of disability inventory: computer adaptive test in children with cerebral palsy. Phys Ther 2017;97:669-76.

139 Ziviani J, Ottenbacher KJ, Shephard K, et al. Concurrent validity of the functional independence measure for children (WeeFIM(TM)) and the pediatric evaluation of disabilities inventory in children with developmental disabilities and acquired brain injuries. Phys Occup Ther Pediatr 2002;21:91-101.

140 Dumas HM, Fragala-Pinkham MA, Haley SM, et al. Computer adaptive test performance in children with and without disabilities: prospective field study of the PEDI-CAT. Disabil Rehabil 2012:34:393-401

141 National Disability Insurance Agency. Providing evidence of disability for children, 2021. Available: https://www.ndis.gov. au/applying-access-ndis/how-apply/information-support-yourrequest/providing-evidence-disability-children [Accessed 3 Mar 2021].

142 Dumas HM, Fragala-Pinkham MA, Rosen EL, et al. Pediatric evaluation of disability inventory computer adaptive test (PEDI-CAT) and Alberta infant motor scale (aims): validity and responsiveness. Phys Ther 2015;95:1559-68.

143 Carter A, Briggs-Gowan M. ITSEA: Infant-Toddler social and emotional assessment. Massachusetts: PsychCorp, 2006.

144 Sanner N, Smith L, Wentzel-Larsen T, et al. Early identification of social-emotional problems: applicability of the Infant-Toddler social emotional assessment (ITSEA) at its lower age limit. Infant Behav Dev 2016;42:69-85.

145 Carter AS, Briggs-Gowan MJ, Jones SM, et al. The Infant-Toddler social and emotional assessment (ITSEA): factor structure, reliability, and validity. J Abnorm Child Psychol 2003;31:495-514.

146 Mclntyre S, Morgan C, Walker K, et al. Cerebral palsy--don't delay. Dev Disabil Res Rev 2011:17:114-29.

147 Surveillance of Cerebral Palsy in Europe. Surveillance of cerebral palsy in Europe: a collaboration of cerebral palsy surveys and registers. surveillance of cerebral palsy in Europe (SCPE). Dev Med Child Neurol 2000;42:816-24.

148 Smithers-Sheedy H, Badawi N, Blair E, et al. What constitutes cerebral palsy in the twenty-first century? Dev Med Child Neurol 2014;56:323-8.

149 Palisano R, Rosenbaum P, Walter S, et al. Development and reliability of a system to classify gross motor function in children with cerebral palsy. Dev Med Child Neurol 1997;39:214-23.

150 Wood E, Rosenbaum P. The gross motor function classification system for cerebral palsy: a study of reliability and stability over time. Dev Med Child Neurol 2000;42:292-6.

151 Oeffinger DJ, Tylkowski CM, Rayens MK, et al. Gross motor function classification system and outcome tools for assessing ambulatory cerebral palsy: a multicenter study. Dev Med Child Neurol 2004:46:311-9.

152 Palisano RJ, Rosenbaum P, Bartlett D, et al. Content validity of the expanded and revised gross motor function classification system. Dev Med Child Neurol 2008;50:744-50.

153 Gorter JW, Rosenbaum PL, Hanna SE, et al. Limb distribution, motor impairment, and functional classification of cerebral palsy. Dev Med Child Neurol 2004;46:461-7.

154 Eliasson A-C, Ullenhag A, Wahlström U, et al. Mini-MACS: development of the manual ability classification system for children younger than 4 years of age with signs of cerebral palsy. Dev Med Child Neurol 2017:59:72-8.

155 Bryson S, Zwaigenbaum L. Autism observation scale for infants. In: Patel V, Preedy V, Martin C, eds. Comprehensive guide to autism. 1st ED. New York: Springer-Verlag, 2014: 299-310.

156 Georgiades S, Szatmari P, Zwaigenbaum L, et al. A prospective study of autistic-like traits in unaffected siblings of probands with autism spectrum disorder. JAMA Psychiatry 2013;70:42-8.

157 Bradley KA, Kivlahan DR, Williams EC. Brief approaches to alcohol screening: practical alternatives for primary care. J Gen Intern Med 2009;24:881.

158 O'Leary CM, Bower C, Zubrick SR, et al. A new method of prenatal alcohol classification accounting for dose, pattern and timing of 
exposure: improving our ability to examine fetal effects from low to moderate alcohol. J Epidemiol Community Health 2010;64:956-62.

159 Hemingway SJA, Bledsoe JM, Brooks A, et al. Comparison of the 4-digit code, Canadian 2015, Australian 2016 and Hoyme 2016 fetal alcohol spectrum disorder diagnostic guidelines. Adv Pediatr Res 2019;6:31.

160 Tsang TW, Laing-Aiken Z, Latimer J, et al. Digital assessment of the fetal alcohol syndrome facial phenotype: reliability and agreement study. BMJ Paediatr Open 2017;1:e000137.

161 Lovibond SH, Lovibond PF. Manual for the depression anxiety stress scales. 2nd edn. Sydney: Psychology Foundation, 1995.

162 Lovibond PF, Lovibond SH. The structure of negative emotional states: comparison of the depression anxiety stress scales (DASS) with the Beck depression and anxiety inventories. Behav Res Ther 1995:33:335-43.

163 Brown TA, Chorpita BF, Korotitsch W, et al. Psychometric properties of the depression anxiety stress scales (DASS) in clinical samples. Behav Res Ther 1997;35:79-89.
164 Yelland J, Sutherland G, Brown SJ. Postpartum anxiety, depression and social health: findings from a population-based survey of Australian women. BMC Public Health 2010;10:10.1186/1471-245810-771.

165 Bush K, Kivlahan DR, McDonell MB. The audit alcohol consumption questions (AUDIT-C): an effective brief screening test for problem drinking. Arch Intern Med 1998;158:10.1001/archin te.158.16.1789:1789-95.

166 Spittle AJ, Ferretti C, Anderson PJ, et al. Improving the outcome of infants born at $<30$ weeks' gestation--a randomized controlled trial of preventative care at home. BMC Pediatr 2009;9:10.1186/1471-2431-9-73.

167 Roberts G, Howard K, Spittle AJ, et al. Rates of early intervention services in very preterm children with developmental disabilities at age 2 years. J Paediatr Child Health 2008;44:276-80.

168 StataCorp LLC. Stata statistical software: release 16 College Station, TX: StataCorp LLC, 2019. Available: https://www.stata. com/ [Accessed 10 Dec 2020]. 